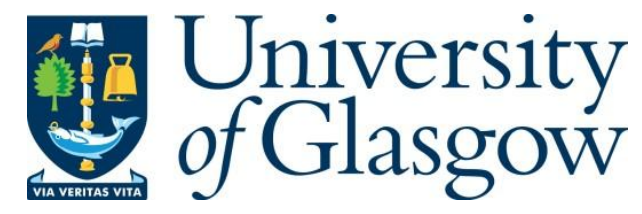

Zare-Behtash, H., Gongora-Orozco, N., Kontis, K., and Holder,

S.J. (2009) Application of novel pressure-sensitive paint formulations for the surface flow mapping of high speed jets. Experimental Thermal and Fluid Science, 33 (5). pp. 852-864. ISSN 0894-

1777 (doi:10.1016/j.expthermflusci.2009.03.002)

Copyright @ 2009 Elsevier

A copy can be downloaded for personal non-commercial research or study, without prior permission or charge

Content must not be changed in any way or reproduced in any format or medium without the formal permission of the copyright holder(s)

When referring to this work, full bibliographic details must be given

http://eprints.gla.ac.uk/84647/

Deposited on: 06 January 2014

Enlighten - Research publications by members of the University of Glasgow http://eprints.gla.ac.uk 


\section{Application of Novel Pressure-Sensitive Paint Formulations for the Surface Flow Mapping of High Speed Jets}

\section{H. Zare-Behtash, N. Gongora-Orozco and K. Kontis}

The University of Manchester, Aero-Physics Laboratory, School of MACE, George Begg Building, Sackville Street, Manchester, M60 1QD, UK

E-mail: h.zare-behtash@postgrad.manchester.ac.uk

\section{S. J. Holder}

The University of Kent, Functional Materials Group, School of Physical Sciences, Canterbury, Kent, CT2 $7 \mathrm{NH}, \mathrm{UK}$

\section{Abstract}

The principle of pressure-sensitive paints (PSPs) is based upon excitation of the luminophore molecules at a certain wavelength and the emission of this absorbed energy at a higher wavelength. By isolating these two wavelengths we insure that the results obtained are not affected by any background radiation. Various international research groups, such as: the Central Aero-Hydrodynamic Institute (Russia), the University of Washington, NASA Ames, Boeing and McDonnell Douglas (USA), have developed their PSP formulations and some are commercially available.

Two paints, which have been developed in-house at the Aero-Physics Laboratory (APL) at the University of Manchester, are studied here. One formulation uses hydrochloric acid (PSP1-HCl) and the other acetone as the solvent (PSP2-Ace). The current study employs the well known schlieren photography technique together with the relatively new PSP method, with comparison to discrete measurements, to examine the flow through a two-dimensional air-ejector system and examines the efficacy of the PSP formulations in providing an accurate global pressure field of the aforementioned setup. Detailed analysis of the errors and drawbacks involved in PSP measurements along with possible solutions to overcome them are also presented. Fully expanded jet Mach numbers in the range of $0.52 \leq M_{j} \leq 1.36$ were examined. 


\section{Nomenclature}

$A \quad$ Area $\left[\mathrm{m}^{2}\right]$

$A(T), B(T), C(T)$ Polynomial coefficients

$b \quad$ Pressure sensitivity of $\operatorname{PSP}\left(=\Delta\left(I_{\text {ref }} / I\right) / \Delta P\right)$

$H_{\text {inlet }} \quad$ Inlet height of the test section [mm]

I Light intensity $\left[\mathrm{mWm}^{2} \mathrm{~nm}^{-1}\right]$

$I_{o} \quad$ Luminescence intensity when oxygen concentration is zero $\left[\mathrm{mWm}^{2} \mathrm{~nm}^{-1}\right]$

$I_{e} \quad$ Intensity of excitation light source $\left[\mathrm{mWm}^{2} \mathrm{~nm}^{-1}\right]$

$I_{\text {ref }} \quad$ Luminescent intensity at known constant reference pressure $\left[\mathrm{mWm}^{2} \mathrm{~nm}^{-1}\right]$

$K_{S V} \quad$ Stern-Volmer constant

Mach number, Molar mass [g/mol]

$M_{j} \quad$ Fully expanded jet Mach number

$N \quad$ Number of photoelectrons detected by CCD camera

$N_{\max } \quad$ Maximum number of photoelectrons detected by CCD camera (full-well capacity)

$\mathrm{O}_{2} \quad$ Oxygen

$P \quad$ Pressure [bar]

$P_{\infty} \quad$ Free-stream static pressure [bar]

$P_{o} \quad$ Total pressure [bar]

$P_{b} \quad$ Back pressure [bar]

$P_{\text {inlet }} \quad$ Inlet pressure supplied through the test section [bar]

$P_{j} \quad$ Jet static pressure at nozzle exit [bar]

$P_{\min } \quad$ Minimum pressure on model surface [bar]

$P_{\text {ref }} \quad$ Reference pressure [bar]

$S \quad$ Henry's solubility constant

Temperature $[\mathrm{K}]$

$u \quad$ Velocity $[\mathrm{m} / \mathrm{s}]$

$X \quad$ Horizontal distance [mm]

$\gamma \quad$ Specific heat ratio $\left(=C_{p} / C_{v}\right)$, taken as 1.4

$\delta_{N} \quad$ Relative error of luminescence intensity

$\delta_{p} \quad$ Relative error of pressure

$\lambda_{e} \quad$ Wavelength of excitation light source [nm]

$\lambda_{\max } \quad$ Wavelength of maximum luminescence [nm] 


\section{Introduction}

\subsection{Pressure-Sensitive Paint (PSP)}

Pressure-sensitive paints (PSPs) have become useful tools to augment conventional pressure taps in measuring the surface pressure distribution of aerodynamic components $[1,2,3,4]$ not only in conventional supersonic flows [5, 6] but also at the micron scale [7]. PSP offers the advantage of non-intrusive global mapping of the surface pressure. The PSP technique is well established in external aerodynamics. However, in internal flows in narrow channels, turbo-machinery and high-speed flow applications there are still unsolved problems complicating the PSP use, accuracy, and reliability [8].

A PSP consists of a dispersion of oxygen-sensitive photoluminescent probe molecules in an oxygen permeable binder layer. An excitation light source of wavelength $\lambda_{e}$ and intensity $I_{e}$ is used to promote molecules to an excited energy state $[9,10]$. There are two major mechanisms for an oxygen sensitive photoluminescent molecule to return to the ground state: (i) the emission of light at a range of wavelengths (maximum absorption $=\lambda_{\max }$ ) with a strong quantum yield of emitted light (intensity $=I$ ); (ii) the transfer of energy by collision with an oxygen molecule, a process called dynamic or oxygen quenching, resulting in a decrease in intensity of the emitted light from the photoluminescent molecules and a reduction in the lifetime of the excited state (Figure 1). The difference in intensities between the photoluminescent molecules at varying concentrations of $\mathrm{O}_{2}$ gives rise to the PSP method whereby luminescence can be used to determine pressure in the immediate environment.

A typical PSP system requires an illumination source for the PSP excitation, a photodetector for acquiring images, and a data reduction tool to process the PSP images into pressure maps through the use of a calibration curve [11]. The luminescent intensity, $I$, is related to oxygen concentration, by the Stern-Volmer equation,

$\frac{I_{o}}{I}=1+\left[O_{2}\right] \times K_{S V}(T)$

where $I_{o}$ is the luminescent intensity when oxygen concentration is zero, $\left[\mathrm{O}_{2}\right]$ is the oxygen concentration, $T$ is the temperature, and $K_{S V}$ is the Stern-Volmer constant. The partial pressure of oxygen, in air, is $21 \%$ of the air pressure; therefore Eq. (1) can be expressed in terms of air pressure, $P$, as follows,

$\frac{I_{o}}{I}=1+0.21 \times P \times S \times K_{S V}(T)$

letting $K=0.21 K_{S V} S$, where $S$ is Henry's solubility constant, then Eq. (2) can be simplified to,

$\frac{I_{o}}{I}=1+P \times K(T)$ 
Equation (3) is usually normalised by the luminescent intensity, $I_{r e f}$, at a known constant reference pressure $P_{\text {ref }}$, under wind-off (no flow) conditions. Therefore the Stern-Volmer relation can be adapted for aerodynamic testing to,

$$
\frac{I_{r e f}}{I}=A(T)+B(T)\left(\frac{P}{P_{r e f}}\right)
$$

where $A(T)$ and $B(T)$ are defined as,

$$
\begin{aligned}
A(T) & =\frac{1}{1+K(T) P_{r e f}} \\
B(T) & =\frac{K(T) P_{r e f}}{1+K(T) P_{r e f}} .
\end{aligned}
$$

In this way $I_{o}$ is eliminated from Eq. (4) allowing for local differences in variables such as paint thickness and luminophore concentration to be accounted for. A more general expression is as follows [12],

$$
\frac{I_{r e f}}{I}=A(T)+B(T)\left(\frac{P}{P_{r e f}}\right)+C(T)\left(\frac{P}{P_{r e f}}\right)^{2}+\ldots
$$

Coefficients $A(T), B(T)$ and $C(T)$ can be determined by either a-priori or in-situ calibration; these coefficients are unique for each temperature. Although from Henry's law that the concentration of oxygen in the binder is linearly dependent on the pressure over the surface for some coatings and conditions, the relationship between the oxygen concentration and the pressure is nonlinear [9], resulting in a nonlinear relationship between the relative intensities and relative pressures.

\subsection{Supersonic Jets}

There are many physical problems which depend for their proper resolution upon knowledge of the behaviour of a jet issuing from an orifice with a velocity greater than the local velocity of sound [13]. Supersonic underexpanded free jets are found in many applications involving jet and rocket propulsion, thrust vectoring, fuel injectors for supersonic combustion, gas stream from a gun and etc. Studies have shown that the major structure of supersonic jets is determined by nozzle pressure ratio and nozzle configuration $[14,15]$.

One way of incorporating the benefits of air breathing into rocket-based launch vehicles is through the use of an ejector system. Ejectors are fluid pumps that are used to entrain secondary flows using a primary flow. For propulsion applications, this entrainment can augment thrust compared to that generated by the primary flow alone and thereby increase performance. Of course high thrust augmentation is only achievable once the gas- 
dynamics and the flow interactions are understood. This idea is central to the development of rocket-based combined cycle (RBCC) engines, in which it is the ejector effect that is primarily responsible for any increased performance over traditional rocket systems during the initial phases of launch [16].

Information about the fluid dynamic quantities of these jets has compelled comparisons of CFD results with schlieren visualisation which is qualitative at best. Insertion of any intrusive probe, such as static and total pressure tubes or a hot-wire probe, changes the shock structures present in these flows significantly [17].

\subsection{Objective of Current Study}

In this paper we will compare and contrast certain characteristics (UV-vis absorption, fluorescence, molecular composition and surface morphology) of two PSP formulations, namely PSP1-HCl and PSP2-Ace, both based on Ruthenium(III) bathophenanthroline perchlorate ( $\mathrm{Ru}(\mathrm{III})$ bathphen) in a silica-gel binder. The PSP2-Ace formulation is a novel approach to film preparation in avoiding the use of a catalytic agent to cross-link the silica-gel, relying instead on atmospheric moisture; it was hoped that this would lead to 'softer' and more permeable silica films and increased $\mathrm{O}_{2}$ and therefore pressure, sensitivity. For the first time differences between the morphologies of the film as observed by scanning electron microscopy (SEM) analysis were also correlated with static and dynamic measurements of air-flow. Significant differences were observed between these formulations in terms of their film morphologies and their oxygen and temperature sensitivity and hence suitability as PSPs.

Unlike the PSP2-Ace formulation, PSP1-HCl exhibits relatively low temperature sensitivity; temperature sensitivity has long been an intrinsic drawback of the PSP technique, forcing researchers to use a combination of PSP along with a Temperature-Sensitive Paint (TSP) to correct for temperature variations along the model surface. Combined with the in-situ calibration procedure we aim to provide a more accurate measurement of the surface pressure of a two-dimensional supersonic air ejector system. Comparison with conventional schlieren photography and discrete pressure measurements has corroborated the qualitative and quantitative capabilities of our PSP formulation. The detail in which the complex shock structures present in the flow have been captured is also quite good. 


\section{Experimental}

\subsection{PSP Formulations and Application}

Silica prepared by the sol-gel technique acts as a good binder for oxygen-sensitive luminophores. Silica gels prepared by this technique (typically using an acid- or base-catalysed condensation of tetralkoxysilanes) (i) are thermally robust (and hence exhibit low temperature-dependent viscosity changes), (ii) able to entrap molecular species and (iii) are highly oxygen permeable. Methyl triethoxysilane (MTEOS) was used as the sol-gel precursor since under optimum conditions it creates a smooth coating with good adhesion (in contrast to tetraethyl orthosilicate (TEOS) which develop cracks and peel easily) [18]. Ruthenium bathophenanthroline perchlorate was chosen as the luminophore as it has been repeatedly demonstrated to demonstrate significant oxygen sensitivity in its luminescence [19, 20, 21]. Two formulations were employed, consisting of: Ruthenium, MTEOS, ethanol, acetone and hydrochloric acid. In the first case (PSP1-HCl) aqueous hydrochloric acid $(\mathrm{HCl})$ was employed to act as a rapidly acting catalyst promoting the condensation of Si-O-Si bonds and the elimination of methanol. $\mathrm{HCl}$ concentrations of $\sim 0.10-0.125 \mathrm{M}$ result in smooth coatings and good adhesion to substrates [18]. In the second case (PSP2-Ace) acetone was employed in place of the $\mathrm{HCl}$; the rationale being that by eliminating a catalyst the silica Si-O network would develop more slowly (essentially catalysed by the hydrolysis of atmospheric oxygen) and lead to a less homogenous silica gel (in terms of surface roughness and porosity) that would lead to higher oxygen permeability and therefore response times of luminescent quenching.

For application the paint components (MTEOS, $\mathrm{Ru}(\mathrm{III})$, ethanol, $\mathrm{HCl}$ or Ace) were rapidly mixed and applied immediately by spray-coating (from a spray gun) the substrate under study. A number of coats were applied (typically 9) until the colour due to the luminophore (yellow) was clearly visible to the naked eye.

Cut-glass slides $(25 \times 25 \times 1 \mathrm{~mm})$ pre-coated with a matte white primer coat were used as the substrates in static calibration tests. The substrates used in supersonic measurements are described below.

\subsection{A-Priori (Static) Calibration Chamber}

A-priori, or as referred to in some text as static calibration, was employed to determine the characteristics of the luminophore sensor within the two different recipes. This was carried out in a pressure/temperature controlled chamber shown in Figure 2. The pressure was monitored with a Honeywell 24PC Series Pressure Sensor and varied in the range between 0 and 4.5bar. Using a peltier heater/cooler manufactured by Greenweld, the temperature of the PSP sample could be controlled. The peltier could produce sub-zero temperatures as low as $258 \mathrm{~K}$, with a maximum working temperature of $343 \mathrm{~K}$, and the dimensions were $30 \times$ 
$30 \times 4.7 \mathrm{~mm}$. A $k$-type thermocouple mounted on the surface of the PSP sample allowed for the continuous monitoring of the surface temperature.

A pair of light emitting diode (LED) panels with peak wavelength of 470nm were designed and manufactured to allow for large surfaces to be illuminated. Each LED panel comprised of $13 \times 10$ LEDs. The luminescent emission was captured by a CCD camera (LaVision Image Intense). A pair of LED panels were employed in order that in any setup the camera could be positioned normal to the test section with an LED panel exciting the PSP from each side, leading to uniform illumination. The main advantage of placing the camera normal to the test section is that it reduces the danger of surface contamination due to internal reflections [8].

Before each experiment the surface with the PSP coat was replaced by a sheet covered in text and the camera focus was adjusted until a sharp image was acquired. To focus the camera, the camera was run in continuous mode with an exposure time similar to that of acquiring images through the experiments. The triggering of the camera recording could be done either manually or via an external TTL signal.

The detection of light from sources other than the PSP coating leads to errors in the measurement and reduction in accuracy. To prevent this, the use of optical filters over the detector and excitation sources was required to ensure the wavelength of the excitation illumination does not overlap with that of the luminescence emission. Optical filtering of the excitation sources allows passage of light at the absorption wavelength of the coating but prevents the transmission of light at the luminescence wavelengths that would contaminate the coating emission seen by the detector. Such filtering was necessary because all the excitation sources used in PSP, with the exception of lasers, produce light over a broad wavelength band that covers not only the PSP absorption wavelength but also the emission wavelength (see Figure 8). Both paints are excited at wavelengths between $400-500 \mathrm{~nm}$ and emit luminescence at $550-650 \mathrm{~nm}$. A combination of two filters was used to capture the emitted light. The first, an orange long pass filter, only allowing the transmission of light with $\lambda>550 \mathrm{~nm}$ and the second filter was an Infra-Red (IR) cut-off filter, preventing the transmission of light with $\lambda>700 \mathrm{~nm}$.

By taking the ratio of wind-off to wind-on (with flow) images the relation between luminescence intensity and pressure was obtained at a constant temperature. The coefficients obtained from plotting intensity ratios against pressure ratio for different temperatures, known as a Stern-Volmer plot, are used to calculate the pressure sensitivity of the PSP and also to convert intensity images to pressure maps. A summation of images was taken for each pressure to increase the signal to noise ratio (SNR).

By taking the ratio of intensities between the wind-off and wind-on images, the effect of paint thickness and luminophore concentration are eliminated [22]. Figure 3 presents a selection of raw images obtained from $a$ priori calibration performed at $294 \mathrm{~K}$ for different pressures (the selection of pressures is random and is 
merely for demonstration purpose). Figure 4 presents the intensity ratios of the same images when using Figure 3(a) obtained at $0.5 \mathrm{bar}$ as the reference. The intensity of the raw images does not appear to be very uniform, the divided images, however, have a much better surface uniformity. Hence when performing calibration of PSP images we consistently used intensity ratios rather than raw intensities.

\subsection{Nozzle-Ejector}

The nozzle shown in Figure 5 was cut from the same piece of steel. This material was used because it has uniform thickness, nominally $19.05 \mathrm{~mm}$, and is stress free and, therefore, does not warp when cut [23]. The nozzle has a contraction ratio of $6: 1$ and a throat height of $9.6 \mathrm{~mm}$. The mixing tube (ejector) side walls were milled to shape and are bolted directly to the outer frame. These mixing tube side walls become parallel to the centre line at the plane of the nozzle exit.

For the PSP experiments, one side of the nozzle is covered using an aluminium plate coated with the PSP while the other side is covered with optical grade perspex. To provide a good seal between the test section and the two joining side walls a thin layer of the Hermatite instant gasket provides a good seal for high pressures and because it is only a very thin layer it does not alter the thickness of the test section, ensuring a truly two-dimensional geometry. A rubber gasket was also thought to be used but because of the very delicate shape and thickness of the nozzle, especially at the exit of the convergent section, this idea was not approved. Figure 5 also shows the location of the pressure tappings on the side wall, marked out with numbers 1 to 5 . For the schlieren experiments, both sides of the test section were covered with optical grade perspex.

The plate which was to be coated with the PSP was initially covered with 2 - 3 layers of RUSTNOT Matt white paint purchased from Farnell, to give a uniform background coating. The plate was allowed to dry and afterwards, the PSP is spray painted using an air-brush and the plate is cured at $343 \mathrm{~K}$ for approximately 7 hours [12]. Once the plate was ready it was fastened on to the nozzle with screws and the side with the perspex was covered with a black piece of cardboard to cut out any light shining on the PSP surface with the exception of just before and during image acquisition.

Before and immediately after each run a wind-off image was captured. Conventionally the wind-off image before is divided by the wind-on image, but due to temperature variations on the model surface, it is common practice to capture a wind-off image after each run. Using the average between the wind-off before and after, reduces bias errors due to long-term drift in the voltage out of the measurement system caused by changes in illumination intensity and photo-degradation of the PSP luminophores [9, 24]. 
Similar to the static calibration, to increase the signal to noise ratio, a summation of images was used for processing in order to mimic the same conditions as the static calibration [25, 26, 27]. The SNR can also be increased by averaging of multiple frames acquired [28, 29]. However, it is impossible to utilise such averaging techniques for truly unsteady pressure measurements [30, 27].

\subsection{Schlieren Apparatus}

High-speed schlieren photography [31, 32] was employed to visualise the flow. The schlieren setup was identical to that used by An et al. [33], Kontis et al. [34] and Kounadis et al. [35] and is shown schematically in Figure 6. The light source is comprised of a 5 Joule argon stabilised pulsed flash system with 625ns duration. The light generated by the flash passes through a plano-convex lens with $75 \mathrm{~mm}$ diameter and $75 \mathrm{~mm}$ focal length. The converged light spot passes through an iris with a variable aperture of 3 - $50 \mathrm{~mm}$ and is finally cut by a slit. The resulting light beam is collimated using a parabolic mirror of $203.3 \mathrm{~mm}$ diameter and $1016 \mathrm{~mm}$ focal length. The collimated light passes through the test region and is then decollimated by another parabolic mirror. The focal point of the second mirror is on a knife edge. The amount of light cut-off could be controlled by the knife edge which affected the sensitivity of the system. The experiments were carried out in dark conditions. The photographs were taken by a large format camera with $4 \times 5$ inch ISO100 black and white Polaroid film. Since the flow was steady, the flash was triggered manually.

\section{PSP Error Analysis}

\subsection{Image Acquisition}

Electrons are created overtime that are independent of the light falling on the CCD detector. These electrons are counted as signal [1]. The dark current signal of the image detector can be unstable; hence a dark image without any light in the test section was acquired before each series of experiments and subtracted from all images of the series.

The most essential source of luminescent intensity error is the photon shot noise $[19,30]$. The shot noise is not an important factor at the lower pressures due to higher luminescence intensity but becomes important at the high pressures due to the inverse proportionality between intensity and pressure $[3,9]$.

An image detector acquires some mean number of photoelectrons proportional to the luminescence intensity $N=\alpha I$ and variance of this mean number caused by the photon shot noise is equal to the square root of this

number $\Delta N=\alpha \Delta I=\sqrt{N}$. The relative error of luminescence intensity in this case is equal to the relative 
error of the acquired number of photoelectrons and is $\delta_{N}=1 / \sqrt{N}$. The minimal error of luminescence intensity is achieved if the number of acquired photoelectrons is equal to the full-well capacity of the image detector $N_{\max }$, thus exposition of the image detector should be adjusted to achieve full-well capacity in a region of minimal pressure on the model surface $P_{\min }$. In this case the relative error of pressure is given by [1]:

$\delta_{p}=\frac{\left(1+b\left(P-P_{r e f}\right)\right) \sqrt{2+b\left(P-P_{r e f}\right)}}{b P \sqrt{N_{\max }} \sqrt{1+b\left(P_{\min }-P_{r e f}\right)}}$

where $b$ is the pressure sensitivity of the paint, and $P_{r e f}$ is the reference pressure. From Eq. (7) the average uncertainty for the static calibration data points, for the range of temperatures tested, was estimated as $4.4 \%$.

The spatial resolution of the technique depends on the minimum pixel size, which can be resolved by the CCD camera. In the present study, it is equivalent to a square of side length $0.0001 \mathrm{~m}$ (or $100 \mu \mathrm{m}$ ) [25].

\subsection{Temperature Errors}

Temperature error is one of the important drawbacks of the optical pressure measurement system [28]. Airflow causes an appearance of temperature distribution on the model surface. If the temperature sensitivity of PSP is not corrected for in complex internal supersonic flows, large localised errors could contaminate the results [29]. In the case of reference image acquisition, additional temperature error appears since the temperature distribution on the model surface during reference image acquisition differs from temperature distribution during wind-on image capture. Therefore, some delay should be made between starting the flow and acquiring the wind-on images, to allow the model surface to reach steady temperature. For the current study once the desired Mach number was set, the flow was left running for approximately 10 seconds before the PSP was exposed to the excitation light. Also, using aluminium as the precursor ensures rapid temperature equilibration.

It is believed that the effects of temperature changes were minimised by the use of in-situ calibration using wall static taps. In a sense, in-situ PSP calibration eliminates the systematic error associated with the temperature effect and the illumination change by absorbing it into the overall fitting error [36]. Also, in continuous flows, errors due to temperature are generally small when wind-on and wind-off images are acquired after the model temperature has stabilised [24], which has been the case for the current study. 


\subsection{Photo-degradation}

The illumination of the luminescent pressure sensor by excitation light initiates various photochemical processes. These processes cause destruction of the luminophore and binder, thus, producing a change in the paint characteristics. The most essential effect of photo-degradation is a decrease of luminescence intensity during illumination by excitation light, producing a calculated pressure higher than the actual pressure.

Since the intensity of the emitted illumination is proportional to the excitation illumination, the source of illumination must be of sufficient power in the absorption spectrum of the PSP coating, have a stable output over time, and must be well ventilated to maintain a constant operating temperature [9]. Before the experiment commenced the LED panels had to be switched on for approximately 5 minutes. This was due to the fluctuation of the power supply. The voltage was set at 30 volts which was the voltage used for the static calibration and monitored throughout the experiments. The LED panels were placed within rigid housings with multiple vents on each side to provide adequate ventilation [37, 38].

At a very high intensity of excitation light, a change of calibration curve caused by saturation can appear. In this case, the calibration coefficients depend not only on pressure and temperature, but also on excitation light intensity. Because the camera was $12 \mathrm{bit}$, it is capable of capturing electrons up to a maximum of 4096 counts $\left(2^{12}\right)$; beyond which image saturation occurs. Excitation light intensity must be restricted to avoid this effect.

For complex models with numerous surfaces such as the test case investigated in the current study, multiple illumination elements are often needed to achieve an adequate coverage of the model surface. To prevent any photo-degradation under ambient light the models covered with the PSP coatings were stored in the dark.

\subsection{Static Calibration}

The accuracy of the static calibration results correspond to the various directly measured physical quantities which affect the precision of the pressure data computed from them [28]. These include: that of the thermocouple and pressure transducer used for calibration, which are in the range of $\pm 0.5 \mathrm{~K}$ and $\pm 250 \mathrm{~Pa}$, depending on the absolute temperature and pressure, respectively [25].

The error due to curve fitting of the calibration data to Eq. (6) was calculated since it plays an important role in the conversion of intensity into pressure maps [11]. Figure 7 represents a curve fitting example for the PSP2-Ace. The points on the graphs are actual data collected from the experiments and the lines show the second order polynomial curve fit. The error was calculated using Matlab by taking each point of the experimental data, regarded as the 'true value,' with the corresponding value from the polynomial and 
finding the difference as a percentage of the true value. For the PSP2-Ace the average error for the cases tested was estimated at $1.2 \%$, and $7.0 \%$ for the PSP1-HCl.

\section{Results and Discussion}

\subsection{Analysis of the Pressure Sensitive Paint (PSP1-HCl and PSP2-Ace) Films}

Acetone was employed as a solvent/diluent in place of $\mathrm{HCl}$ in one of the formulations to monitor the effects of silica-gel on PSP performance. The excitation and emission spectra of thin films of PSP1 and PSP2 coated on quartz-slides were recorded and the spectra that resulted are illustrated in Figure 8. Whereas the excitation spectra are similar, the maximum emission wavelength for the $\mathrm{Ru}(\mathrm{III})$ bathophenanthroline perchlorate in the PSP2-Ace film is clearly shifted towards higher wavelengths $\left(\lambda_{\max }=598 \mathrm{~nm}\right)$ compared to that for the PSP1$\mathrm{HCl}$ film $\left(\lambda_{\max }=586 \mathrm{~nm}\right)$ and furthermore the intensity of the dye in the PSP2-Ace film is reduced (despite being present in the same concentration as in PSP1-HCl). Attenuated total reflection Fourier-transform infrared spectroscopic analysis (ATR FT-IR) of the two films presented in Figure 9 showed clear chemical differences between the two samples. Although the spectrum obtained for the PSP1-HCl is that expected for $\mathrm{Ru}(\mathrm{III})$ bathphen in a silica-gel, that of the PSP2-Ace film displays a less well-developed silica (Si-O) network structure and the presence of significant amounts of acetone. Whereas the less well developed Si-O network is a natural and expected consequence of the absence of the catalyst in the formulation, the presence of acetone is less readily explained. No smell of acetone was detectable and no reduction in the acetone peaks was visible even after several weeks under ambient conditions. Consequently it must be assumed that the acetone is entrapped in the thin film either free or co-ordinated to the $\mathrm{Ru}(\mathrm{III})$. Given the shifted wavelength of the PSP2-Ace emission wavelength compared to that of the PSP1-HCl and that bathochromic shifts and reduced luminescent intensities are commonly observed for dyes in increasingly polar environments (acetone versus silica) [39], it is likely that the acetone is directly co-ordinated to the $\mathrm{Ru}(\mathrm{III})$ bathphen; similar acetone co-ordinated $\mathrm{Ru}$ complexes have been previously isolated and directly observed [40, 41]. Differences between the films were even more apparent by SEM analysis of the surfaces of the thin films cast on glass. SEM images are illustrated in Figure 10. As anticipated the film prepared from acetone (PSP2-Ace) shows considerably more surface roughness than the highly smooth and largely uniform surface of the film prepared from $\mathrm{HCl}(\mathrm{PSP} 1-\mathrm{HCl})$. 


\subsection{Static Calibration and Comparison of the Pressure Sensitivity of PSP Films}

The pressure sensitivity of the PSP defined as $\Delta\left(I_{\text {ref }} / I\right) / \Delta P$ (per bar) is calculated from the Stern-Volmer (S-V) plot. Figures 11 and 12 present the S-V plots for PSP1-HCl and PSP2-Ace, respectively. From visual inspection it is clear that PSP1-HCl has greater pressure sensitivity (deduced from the higher slope of $I_{\text {ref }} I$ vs. $P$ ). PSP2-Ace displays only $9.3 \%$ the sensitivity of PSP1-HCl at $T_{\min }$ and $17.8 \%$ the sensitivity at $T_{\max }$.

It is apparent that the PSP2-Ace film is significantly less sensitive to $\left[\mathrm{O}_{2}\right]$ than the PSP1-HCl film. To a certain extent this is surprising as the rougher surface of the PSP2-Ace film might be expected to lead to an increased response as a result of the increased surface area and hence a relative increase in $\mathrm{O}_{2}$ diffusion might have been expected. However this loss of sensitivity might be attributed to the acetone environment of the $\mathrm{Ru}(\mathrm{III})$ bathphen dye in the PSP2-Ace film. As previously noted acetone would appear to be directly coordinated to the $\mathrm{Ru}(\mathrm{III})$ centre which would require dissociation of the acetone from the metal centre prior to $\mathrm{O}_{2}$ binding this would inevitably lead to a reduction in the sensitivity of the quenching process. It is also possible that acetone is entrapped in the matrix in liquid form and that $\mathrm{O}_{2}$ solubility in, and diffusion rates through, are lower than in the $\mathrm{SiO}_{2}$ matrix. However it is difficult to envisage a structural model where the film is sufficiently impermeable such that the acetone is entrapped and unable to vaporise, but the film is still sufficiently permeable to allow $\mathrm{O}_{2}$ diffusion. In either case the difference in the photoluminescent spectra (a red-shift in the maximum excitation peak and a reduction in the luminescent intensity) between the two films and the concomitant difference in quenching sensitivities strongly supports some form of interaction between acetone and the Ru dye.

\subsection{Accuracy of PSPs}

Figure 13 shows the dynamically calibrated PSP1-HCl and PSP2-Ace pressure profiles along the central axis of the nozzle together with discrete pressure measurements. Whereas the Acetone based PSP exhibits a plethora of fluctuations along its profile, the $\mathrm{HCl}$ based paint has a much more uniform profile. The reason being the difference in surface uniformity discussed earlier and presented in the SEM images of Figure 10. The rms error was obtained by taking the difference between PSP and 'true' static pressure tap values at each of the pressure tap locations and calculating a root mean square (expressed as a percent of the true value) [28, 42]. The rms error was calculated as $3.2 \%$ for PSP1-HCl, and $6.0 \%$ for PSP2-Ace. Evidently the accuracy of the PSP1-HCl is superior to that of PSP2-Ace. 


\subsection{Nozzle Flow}

The inlet pressure was supplied through a regulator and monitored via a transducer placed on the inlet wall of the nozzle (plenum). The plenum to ambient pressure was varied from 1.2 in increments of $0.2 \mathrm{bar}$ up to a pressure ratio of 3.0, with the free stream (ambient) pressure taken as Ibar. This corresponds to a fully expanded Mach number range $0.52 \leq M_{j} \leq 1.36$. The 'fully expanded Mach number,' $M_{j}$, is the ideal Mach number achievable by isentropically expanding the plenum pressure to the ambient value $[15,17,43,44] . M_{j}$ is given as,

$M_{j}=\sqrt{\frac{2}{\gamma-1}\left[\left(\frac{p_{o}}{p_{\infty}}\right)^{\frac{(\gamma-1)}{\gamma}}-1\right]}$

Due to the relatively low Mach number at the nozzle entrance the pressure at the nozzle entrance can be used as the total pressure $P_{o}[45,46]$.

The schlieren photographs of the flow along with the PSP results corresponding to the same inlet conditions are given in Figures 14 and 15. Several flow features can immediately be discerned from the PSP pressure maps, the main one being that the PSP results appear to corroborate the schlieren images in identifying the location of the flow features quite well.

When a jet exhausts from a nozzle into still air, it will undergo a two-dimensional expansion or compression exactly at the nozzle lip dependent only upon whether $P_{j} / P_{\infty}>1$ or $P_{j} / P_{\infty}<1$, respectively [14]. Where $P_{j}$ is the jet static pressure at the nozzle exit and $P_{\infty}$ is the ambient or free-stream static pressure. By slowly increasing the inlet pressure, the local Mach number will increase slightly through the convergent portion of the nozzle, reaching a maximum at the throat. From Figures 14(a) and 14(b) we see the expanding flow exiting the nozzle into the ejector section with quiescent air.

If the pressure difference is further increased, the stronger pressure ratio between the inlet and exit accelerates the flow and the variations of subsonic Mach number and static pressure through the duct will be larger. In the convergent portion of the nozzle, the subsonic flow is accelerated, with the subsonic value of $M$ dictated by the local value of $A / A^{*}$, where the asterisk denotes conditions at sonic speed and $A$ the area of the nozzle inlet, as given by Eq. 9 .

$\left(\frac{A}{A^{*}}\right)^{2}=\frac{1}{M^{2}}\left[\frac{2}{\gamma+1}\left(1+\frac{\gamma-1}{2} M^{2}\right)\right]^{(\gamma+1) /(\gamma-1)}$

At higher inlet pressures, the Mach number at the throat cannot increase beyond $M=1$. This is dictated by Eq. 10. Hence, the flow properties at the throat and indeed throughout the entire subsonic section of the duct become frozen and the mass flow remains constant, corresponding to the choked flow condition. 


$$
\frac{d A}{A}=\left(M^{2}-1\right) \frac{d u}{u}
$$

To determine whether the flow at the exit is sonic or subsonic (the flow at the exit could never be supersonic since the nozzle area does not diverge), the ratio of back pressure to total pressure, $P_{b} / P_{o}$ must be evaluated. This is then compared to the critical pressure ratio, given by:

$$
\frac{P^{*}}{P_{o}}=\left(\frac{2}{\gamma+1}\right)^{\gamma /(\gamma-1)}
$$

which, for air, is 0.528 . If $P_{b} / P_{o}<P * / P_{o}$, the exit pressure is higher than the back pressure, so the exit flow must be sonic. Pressure equilibration is achieved after the exit by a series of expansion waves. If on the other hand $P_{b} / P_{o}>P^{*} / P_{o}$, the flow exits subsonically [45].

At higher Mach numbers, in the convergent portion of the duct nothing happens. However, a lot happens in the divergent portion of the duct. As the plenum pressure is increased beyond the choked flow condition, a series of shock cells form in the jet plume, as visible in schlieren photograph of Figure 14(c) and the PSP map of Figure 14(d). The sharp vertical boundaries at the end of each compression zone correspond to the termination position of each shock in the shear layer [47]. This oblique shock wave pattern is reminiscent of an overexpanded flow with the increase to back pressure taking place across an oblique shock attached to the nozzle exit. In the same figures we see that the primary jet is not symmetrical within the mixing tube and has bent over to run along the upper wall. This flow condition will not be very stable since a slightly lower pressure on one side of the jet will cause it to switch to that side without producing a stabilising pressure decrease at the other side $[15,23]$.

Figures 14(e) and 14(f), however, exhibit properties of underexpanded jets since the flow is capable of additional expansion. An underexpanded jet starts with an expansion process where pressure progressively decreases until the shock compression region is encountered where pressure jumps to a higher value. Equilibrium of the flow takes place across expansion waves outside the duct, since across the expansion fan the pressure decreases and thus the sudden acceleration of the flow is communicated to the surrounding flow [48]. The supersonic flow is decelerated and the wall static pressure increases through a shock train region. The region where the series of shocks in line can be visible by optical observations is referred to as the region of shock train [49]. In an underexpanded jet, shocks are formed by a mechanism of internal reflection of expansion waves into compression waves from the supersonic-subsonic interface, and the subsequent coalescence of the compression waves [50].

The jet which exhausts into the atmosphere from the nozzle exit has a boundary surface which interfaces with the surrounding quiescent gas. The pressure across this boundary must be preserved; hence the jet boundary pressure must equal $P_{\infty}$ along its complete length. Therefore the oblique shock waves visible in Figure 14(c) 
and the expansion waves in Figure 14(e) must reflect from the jet boundary in such a fashion as to preserve the pressure at the boundary downstream of the nozzle exit. This boundary is not a solid surface; rather it is a free boundary which can change in size and direction. Upon collision with a free boundary, incident shocks (compression waves) are reflected as expansion fans which lead to the communication of the high pressure upstream of the incident wave and the free stream pressure behind the expansion fan. The flow is deflected outwards by both the incident and the reflected expansion causing the free boundary to deflect outwards also. This mechanism is responsible for the generation of the shock cells visible in Figures 14 and 15.

Observing the schlieren and PSP images of events taking place in Figure 15, which shows results for higher values of $M_{j}$ than Figure 14, the expansion zones are relatively longer with the pressure within the convergent section continuing to increase. The schlieren photographs of Figures 15(a), 15(c) and 15(e) reveal the increase in the shock cell size with their boundaries better defined. This is also depicted in the PSP images. The shock waves, especially the ones lying further downstream from the nozzle exit, undergo considerable distortion and show some oscillation. The first shock cell, however, does not show any discernible motion, a finding similar to that of Panda [50].

The accuracy of identifying the individual shock cells from the schlieren images tended to decrease slightly as $M_{j}$ increased as a result of decrease in definition caused by the increased turbulence surrounding the jet. Hence, the sensitivity of the schlieren system was reduced in Figure 15.

The acoustic emission from an underexpanded free jet generally has a spectrum that contains very powerful discrete tones called screech. In Figure 15(a) it is observed that a sinuous large-scale turbulent structure crosses the rear edge of the third shock cell. This has been marked with location S1 and S2 being the prominent point (or a sound source) on the sinuous large-scale turbulent structure around the jet. The location of this structure is coincident with the findings of Kweon et al. [15] being at the rear edge of the shock cells. The interaction between these points and the oblique shock cells structures, produce strong acoustic waves which are radiated and propagate upstream. The sound waves stimulate the sensitive thin shear layer near the nozzle lip [50,51,52]. According to Panda [50] the primary reason for the large amplitude shock oscillation is a combination of pressure fluctuations associated with the passage of the large organised structures along the jet shear layer, and the distortion of the supersonic-subsonic interface in the jet shear layer caused by the same organised vortices.

Due to viscous effects, the flow exiting the nozzle entrains air from the surrounding. This is depicted by the reduction in static pressure above and below the nozzle plenum. As $M_{j}$ is increased, the velocity of the entrained fluid also increases. This can be seen by comparing Figures 14(a) and 15(f). 
Examining closely Figures 16(a) and 16(b), which provide close-up of Figures 14(f) and 15(f), we can see how the deflection of the last expansion fan increases relative to the normal with increasing flow Mach number, tending to become parallel to the free stream at higher Mach numbers.

Figure 17 is the pressure profile along the centreline of the nozzle for various inlet pressures. Examining firstly Figure 17(a), for inlet pressures of 1.2bar and 1.4bar the flow exits subsonically. However, at an inlet pressure of 1.6 bar denoted by the red line we observe a small dip and a sharp rise at approximately $X / H_{\text {inlet }}=$ 1.8 which corresponds to the exit of the nozzle. The rise in pressure is due to the presence of a normal shock wave just at the exit of the nozzle. This normal shock is barely visible in Figures 14(a) and 14(b). With increasing pressure up to 2.0bar in Figure 17(a), the shock cell patterns are clearly visible in the pressure profiles. It is between 1.8bar and 2.0bar inlet pressures that the jet changes from being overexpanded to underexpanded. Of course if the profiles were taken over a longer distance downstream, the pressure would be expected to return to that of ambient. Figure 17(b) shows similar plots to Figure 17(a), but at higher inlet pressures. From $X / H_{\text {inlet }}=0-0.5$ which represent the converging section of the nozzle the pressure is almost uniform. From $X / H_{\text {inlet }}=0.5-0.75$ where the nozzle converges further into the uniform area channel, the pressure drops indicating an increase of Mach number according to the area relation given in Eq. (9). Through the constant area section of the nozzle just before the exit $\left(X / H_{\text {inlet }}=0.75-1.2\right)$ the flow accelerates only slightly, however, there is sudden expansion of the flow at the exit of the nozzle shown by the very steep drop in static pressure. This exists for all inlet pressures between 2.2-3.0bar. The rate of flow acceleration is much greater at higher pressures depicted by the higher gradient of the pressure drop between $X / H_{\text {inlet }}=1.2-1.3$. The number of shock cells appears to be constant with increasing inlet pressure, deduced from the peaks present in Figure 17(b). The length of these cells, however, as also observed in the schlieren images of Figures 14 and 15, increases at higher Mach numbers. The profiles of Figure 17(b) show the decay of the shock-cell structures with distance and the asymptotic variation of pressure. This is due to viscous losses and mixing which dissipates the shock cells further downstream from the nozzle exit.

Although the accuracy of the PSP2-Ace formulation in comparison to the discrete measurements appeared to be relatively low, the paint is still able to capture the physical features of the flow. Figure 18 shows a comparison between the PSP images obtained from both $\mathrm{HCl}$ - and Acetone-based paints. Similar to the $\mathrm{HCl}$ based PSP, the Acetone-based PSP is also capable of capturing the number and location of the shock cells. The magnitude of the pressures however, are lower than the $\mathrm{HCl}$ formulation and the discrete measurements. Referring back to Figure 13, it is noticeable that the PSP2-Ace paint is as accurate with relation to the discrete measurements as the PSP1- $\mathrm{HCl}$ for all pressures above 0.75 bar. It only deviates very slightly for low pressures. At lower pressure along the model surface the acceleration of the flow is accompanied by the drop 
in surface temperature. From the S-V plot of Figure 12 it is evident that PSP2-Ace has low pressure sensitivity leading to the inaccurate representation of pressure.

\section{Conclusions}

The present study examined the efficacy of two ruthenium based pressure-sensitive paints, developed at the Aero-Physics Laboratory (APL) at The University of Manchester, in performing quantitative and qualitative measurements on a supersonic jet.

Two paint recipes were developed, namely PSP1-HCl and PSP2-Ace. In the first case (PSP1-HCl) aqueous hydrochloric acid $(\mathrm{HCl})$ was employed to act as a rapidly acting catalyst promoting the condensation of Si-OSi bonds and the elimination of methanol. In the second case (PSPS2-Ace) acetone was employed in place of the $\mathrm{HCl}$; the rationale being that by eliminating a catalyst the silica $\mathrm{Si}-\mathrm{O}$ network would develop more slowly leading to a less homogenous silica gel (in terms of surface roughness and porosity) that would lead to higher oxygen permeability.

Scanning electron microscope (SEM) analysis of the PSP films revealed that, as anticipated, the film prepared from acetone (PSP2-Ace) shows considerably more surface roughness than the highly smooth and largely uniform surface of the film prepared from $\mathrm{HCl}$ (PSP1-HCl).

Both paints are excited at wavelengths between $400-500 \mathrm{~nm}$ and emit luminescence at $575-625 \mathrm{~nm}$. Results of the static calibrations revealed that PSP1-HCl has a considerably higher pressure sensitivity relative to PSP2-Ace. PSP1-HCl has proven not only to provide a more accurate global pressure measurement, compared with PSP2-Ace, but also to pinpoint the physical features of the flow such as the flow shear layer, shock cell structures, and even the location and inclination of the last running expansion fan in the underexpanded flows observed. From the quantitative perspective, the pressures obtained by means of PSP1$\mathrm{HCl}$ provide a good correlation with discrete pressure measurements obtained using pressure transducers. The HCl-based PSP results presented differed by $3.2 \%$ compared to the $6.0 \%$ of the Acetone-based paint. Of course the Acetone-based PSP was also capable of the same visual representation of the flow, and provides an accurate indication of pressure for pressures above 0.75 bar. Therefore, it would appear that by using both paints an accurate pressure map can be constructed (e.g. PSP1- $\mathrm{HCl}$ for regions of flow expansion and PSP2Ace for high pressure surface environments). 


\section{Acknowledgments}

The authors would like to thank the fantastic support of Dr. D. Kounadis and the technical staff of the School of MACE for their continuous efforts, Dr. David Ellis for allowing the use of the facilities in the School of Chemistry. The financial support of the EPSRC is also acknowledged. 


\section{References}

[1] V. Moshasrov, V. Radchenko, S. Fonov, Luminescent pressure sensors in aerodynamic experiments, Central Aerodynamic Institute (TsAGI), 1998.

[2] T. Liu, J.P. Sullivan, Pressure and temperature sensitive paints, Springer-Verlag, 2004.

[3] J.H. Bell, E.T. Schairer, L.A. Hand, R.D. Mehta, Surface pressure measurements using luminescence coatings, Annual Review of Fluid Mechanics 33, (2001) 155-206.

[4] K. Kontis, A review of some current research on pressure sensitive paint and thermographic phosphor techniques, The Aeronautical Journal 111, (2007) 495-508.

[5] B.G. McLachlan, J.H. Bell, H. Park, R.A. Kennelly, J.A. Schreiner, S.C. Smith, J.M. Strong, J. Gallery, M. Gouterman, Pressure-sensitive paint measurements on a supersonic high-sweep oblique wind model, Journal of Aircraft 32, (1995) 217-227.

[6] S. Bjorge, F. Reeder, C. Subramanian, J. Crafton, S. Fonov, Flow around an object projected into a supersonic freestream, AIAA Journal 43, (2005) 1465-1475.

[7] H. Nagai, R. Naraoka, K. Sawada, K. Asai, PSP measurement of pressure distribution in a supersonic micronozzle, 46 ${ }^{\text {th }}$ AIAA Aerospace Sciences Meeting and Exhibit, Reno Nevada, AIAA-2006-1046, 2006.

[8] J. Lepicovsky, T.J. Bencic, Use of pressure-sensitive paint for diagnostics in turbo-machinery flows with shocks, Experiments in Fluids 33, (2002) 531-538.

[9] B.F. Carroll, J.D. Abbitt, E.W. Lucas, M.J. Morris, Step response of pressure-sensitive paints, AIAA Journal 34, (1996) 521-526.

[10] H. Sakaue, S. Matsumara, S.P. Schneider, J.P. Sullivan, Anodized aluminium pressure sensitive paint for short duration testing, 22 ${ }^{\text {nd }}$ AIAA Ground Testing Conference, AIAA-2002-2908, 2002.

[11] H. Sakaue, J.W. Gregory, J.P. Sullivan, Porous pressure-sensitive paint for characterizing unsteady flowfields, AIAA Journal 40, (2000) 1094-1098.

[12] C. Wong, M. Amir, C. Lada, K. Kontis, Molecular image sensing for pressure and temperature surface mapping of aerodynamic flows, $42^{\text {nd }}$ AIAA Aerospace Sciences Meeting and Exhibit, AIAA-2004-598, 2004.

[13] D.C. Pack, On the formation of shock-waves in supersonic gas jets, Quarterly Journal of Mechanics and Applied Mathematics 1, (1948) 1-17.

[14] E.S. Love, C.E. Grigsby, L.P. Lee, M.J. Woodling, Experimental and theoretical studies of axisymmetric free jets, NASA technical report, NASA TR R-6, 1959.

[15] Y-H. Kweon, Y. Miyazato, T. Aoki, H-D. Kim, Experimental investigation of nozzle exit reflector effect on supersonic jet, Shock Waves 15, (2006) 229-239. 
[16] J. Etele, B. Parent, J.P. Sislian, Analysis of increased compression through area constriction on ejectorrocket performance, Journal of Spacecraft and Rockets 44, (2007) 355-364.

[17] J. Panda, R.G. Seasholtz, Measurement of shock structure and shock-vortex interaction in underexpanded jets using Rayleigh scattering, Physics of Fluids 11, (1999) 3761-3777.

[18] B.J. Basu, Optical oxygen sensing based on luminescence quenching of platinum porphyrin dyes doped in ormosil coatings, Sensors and Actuators B 123, (2007) 568-577.

[19] J.P. Hubner, B.F. Carroll, K.S. Schanze, H.F. Ji, Pressure-sensitive paint measurements in a shock tube, Experiments in Fluids 28, (2000) 21-28.

[20] G. O’keeffe, B.D. MacCraith, A.K. McEvoy, C.M. McDonagh, J.F. McGilp, Development of a LEDbased phase fluorimetric oxygen sensor using evanescent wave excitation of a sol-gel immobilized dye, Sensors and Actuators B 29, (1995) 226-230.

[21] Y. Tang, E.C. Tehan, Z. Tao, F.V. Bright, Sol-gel-derived sensor materials that yield linear calibration plots, high sensitivity, and long-term stability, Analytical Chemistry 75, (2003) 2407-2413.

[22] B.G. McLachlan, J.H. Bell, H. Park, R.A. Kennelly, J.A. Schreiner, S.C. Smith, J.M Strong, J. Gallery, M. Gouterman, Pressure-sensitive paint measurements on a supersonic high-sweep oblique wing model, Journal of Aircraft 32, (1995) 217-227.

[23] V.A. Eustace, A study of two-dimensional supersonic air ejector systems, PhD thesis, The University of Manchester Institute of Science and Technology, 1969.

[24] C. Raju, P.R. Viswanath, Pressure-sensitive paint measurements in a blowdown wind tunnel, Journal of Aircraft 42, (2005) 908-915.

[25] K. Kontis, C. Lada, H. Zare-Behtash, Effect of dimples on glancing shock wave turbulent boundary layer interactions, Shock Waves Journal 17, (2007) 323-335.

[26] Y. Sakamura, M. Matsumoto, T. Suzuki, High frame-rate imaging of surface pressure distribution using a porous pressure-sensitive paint, Measurement Science and Technology 16, (2005) 759-765.

[27] E.T. Schairer, Optimum thickness of pressure-sensitive paint for unsteady measurements, AIAA Journal 40, (2002) 2312-2318.

[28] M. Sajben, Uncertainty estimates for pressure sensitive paint measurements, AIAA Journal 34, (1993) 2105-2110.

[29] R.R. Taghavi, G. Raman, T.J. Bencic, Mixer-ejector wall pressure and temperature measurements based on photoluminescence, AIAA Journal 40, (2002) 745-750.

[30] J.P. Hubner, B.F. Carroll, K.S. Schanze, H.F. Ji, M.S. Holden, Temperature- and pressure-sensitive paint measurements in short-duration hypersonic flow, AIAA Journal 39, (2001) 654-659. 
[31] G. Settles G, Schlieren and shadowgraph techniques: visualizing phenomena in transparent media, Springer-Verlag, 2001.

[32] W. Merzkirch, Flow visualization, Academic Press Inc., 1974.

[33] R. An, K. Kontis, J.A. Edwards, Compressible vortex-ring interaction studies with a number of generic body configurations, 43 ${ }^{\text {rd }}$ AIAA Aerospace Sciences Meeting and Exhibit, AIAA-2005-1044, 2005.

[34] K. Kontis, R. An, J.A. Edwards, Compressible vortex-ring interaction studies with a number of generic body configurations, AIAA Journal 44, (2006) 2962-2978.

[35] D. Kounadis, R. An, H. Zare-Behtash, K. Kontis, J.A. Edwards, Head-on interaction of shock waves and vortex rings with solid and perforated walls, $36^{\text {th }}$ AIAA Fluid Dynamics Conference and Exhibit, AIAA2006-2875, 2006.

[36] T. Liu, J.P. Sullivan, In situ calibration uncertainty of pressure-sensitive paint, AIAA Journal 41, (2003) 2300-2302.

[37] H. Zare-Behtash, N. Gongora, C. Lada, D. Kounadis, K. Kontis, Application of pressure-sensitive paints in high-speed flows, $26^{\text {th }}$ International Symposium on Shock Waves (ISSW26), Göttingen, Germany, Paper 2341, 2007.

[38] H. Zare-Behtash, N. Gongora, C. Lada, K. Kontis, Application of pressure-sensitive paints to unsteady and high-speed flows, 16th Australian Fluid Mechanics Conference (AFMC), Queensland, Australia, (2007) 466-470.

[39] R. B. Nair, B. M. Cullum, C. J. Murphy, Optical properties of $[R u(p h e n) d p p z]$ as a function of nonaqueous environment, Inorganic Chemistry 36, (1997) 962-965.

[40] S. M. Stevens, R. C. Dunbar, W. D. Price, M. Sena, C. H. Watson, L. S. Nichols, J. M. Riveros, D. E. Richardson, J. R. Eyler, Blackbody infrared radiative dissociation of partially solvated tris $\left(2,2^{6}-\right.$ bipyridine)ruthenium(II) complex ions, The Journal of Physical Chemistry A 106, (2002) 9686-9694.

[41] J. A. Rusanova, S. Decurtins, E. B. Rusanov, H. S. Evans, Crystal structure of bis(2,2ф-bipyridine$N, N \phi)$-[1,10-phenanthroline-5,6-dione]ruthenium(II) dihexafluorophosphate acetone solvate, $\left[\mathrm{Ru}\left(\mathrm{C}_{10} \mathrm{H}_{8} \mathrm{~N}_{2}\right)_{2}\left(\mathrm{C}_{12} \mathrm{H}_{6} \mathrm{~N}_{2} \mathrm{O}_{2}\right)\right]\left[\mathrm{PF}_{6}\right]_{2} \cdot 1.75\left(\mathrm{CH}_{3}\right)_{2} \mathrm{CO}$, Zeitschrift Fur Kristallographie-New Crystal Structures 217, (2002) 571-574.

[42] R. Taghavi, G. Raman, T. Bencic, Pressure sensitive paint demonstrates relationship between ejector wall pressure and aerodynamic performance, Experiments in Fluids 26, (1999) 481-487.

[43] K. Matsuo, K. Sasaguchi, K. Tasaki, H. Mochizuki, Investigation of supersonic air ejectors (Part 1. Performance in the case of zero-secondary flow), The Japan Society of Mechanical Engineers 24, (1981) 2090-2097.

[44] F.M. White, Fluid Mechanics, McGraw-Hill, 2003. 
[45] C.T. Crowe, D.F. Elger, J.A. Roberson, Engineering fluid mechanics, John Wiley \& Sons, 2001.

[46] J.D. Anderson, Modern compressible flow, with historical perspective, McGraw-Hill Inc., 1990.

[47] J. Panda, An experimental investigation of screech noise generation, Journal of Fluid Mechanics 378, (1999) 71-96.

[48] J.J. Bradley, Shock waves in chemistry and physics, Methuen \& Co, Ltd., 1962.

[49] K. Matsuo, Y. Miazato, H-D. Kim, Shock train and pseudo-shock phenomena in internal gas flows, Progress in Aerospace Sciences 35, (1999) 33-100.

[50] J. Panda, Shock oscillation in underexpanded screeching jets, Journal of Fluid Mechanics 363, (1998) 173-198.

[51] Y. Umeda, R. Ishii, Existence of Mach cones and helical vortical structures around the underexpanded circular jet in the helical oscillation mode, Journal of the Acoustical Society of America 112, (2002) 99-107. [52] Y. Umeda, R. Ishii, On the sound sources of screech tones radiated from choked circular jets, Journal of the Acoustical Society of America 110, (2001) 1845-1858. 


\section{Excitation light}

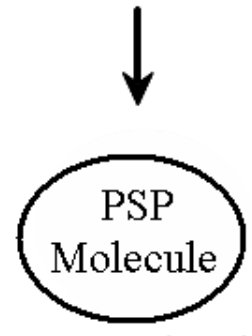

Dye molecule absorbs light

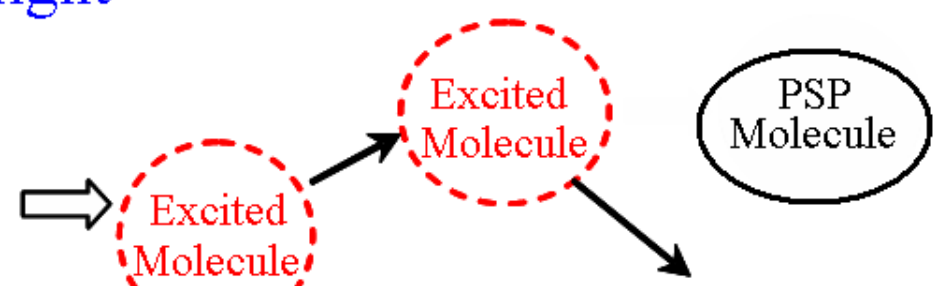

1) Luminescent Emission

\section{Becomes}

Activated

2) Collision quenching by Oxygen molecule

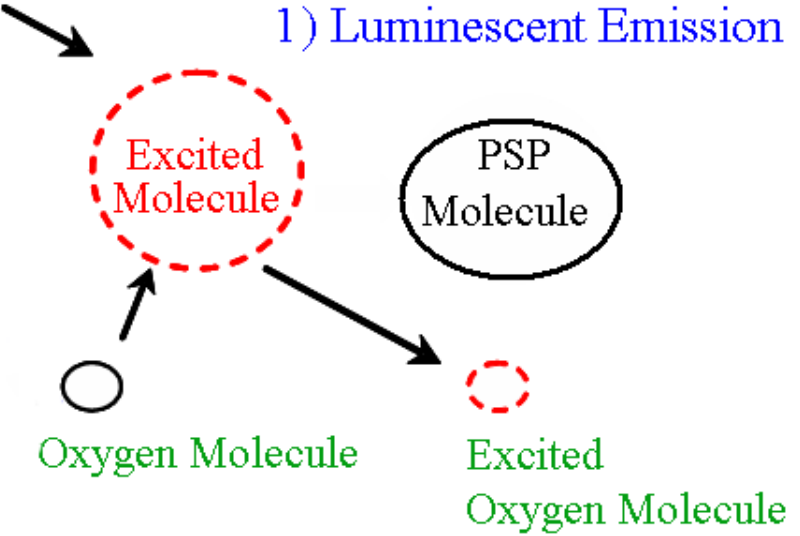

Figure 1. Process of PSP molecule excitation and luminescence.

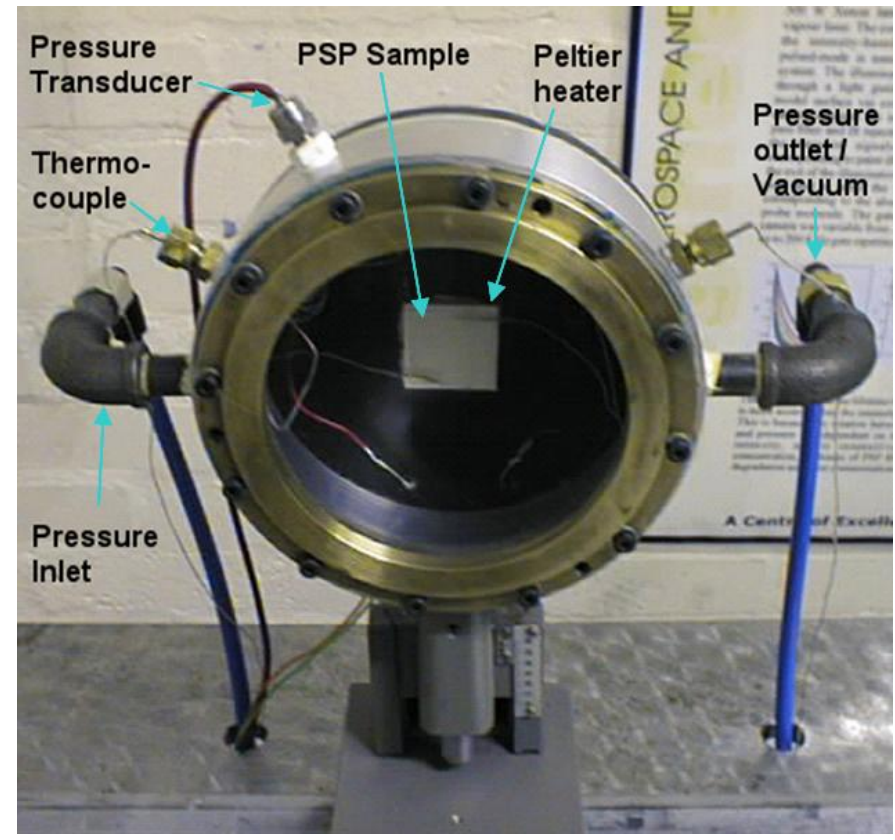

Figure 2. A-priori (static) calibration chamber. 


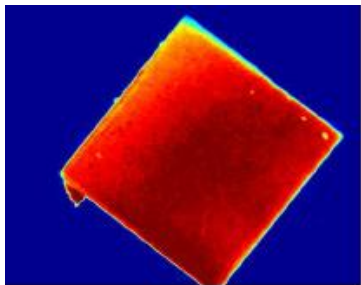

(a)

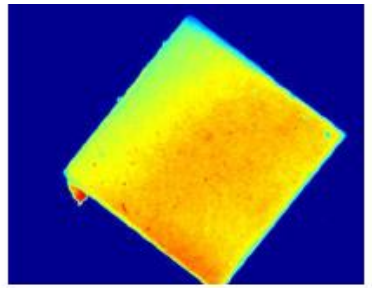

(b)

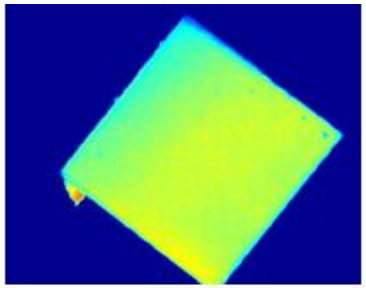

(c)

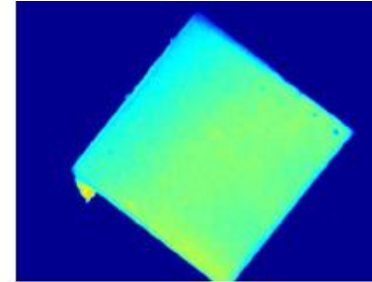

(d)

Figure 3. Raw images obtained from static calibration at pressures: (a) $P=0.5$ bar, (b) $P=1.3$ bar, (c) $P=1.9$ bar, (d) $P=$ 2.5 bar.

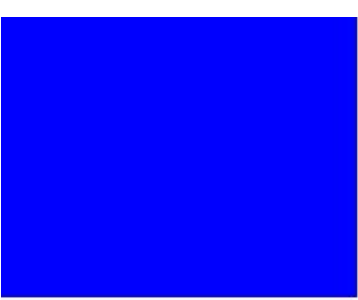

(a)

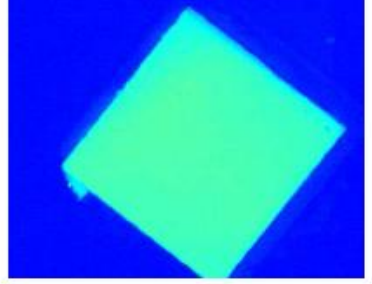

(b)

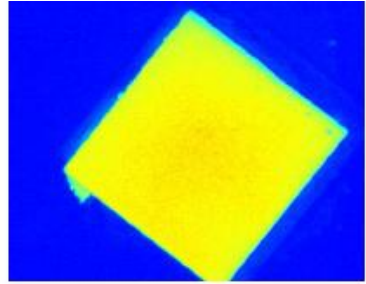

(c)

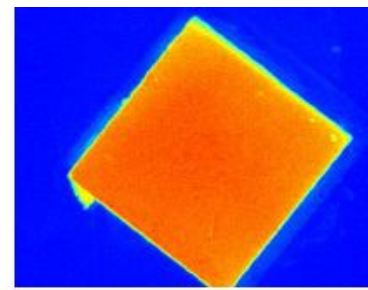

(d)

Figure 4. Results of dividing all the images in Fig. 3 by Fig. 3(a), (a) $P=0.5$ bar, (b) $P=1.3$ bar, (c) $P=1.9$ bar, (d) $P=2.5$ bar.

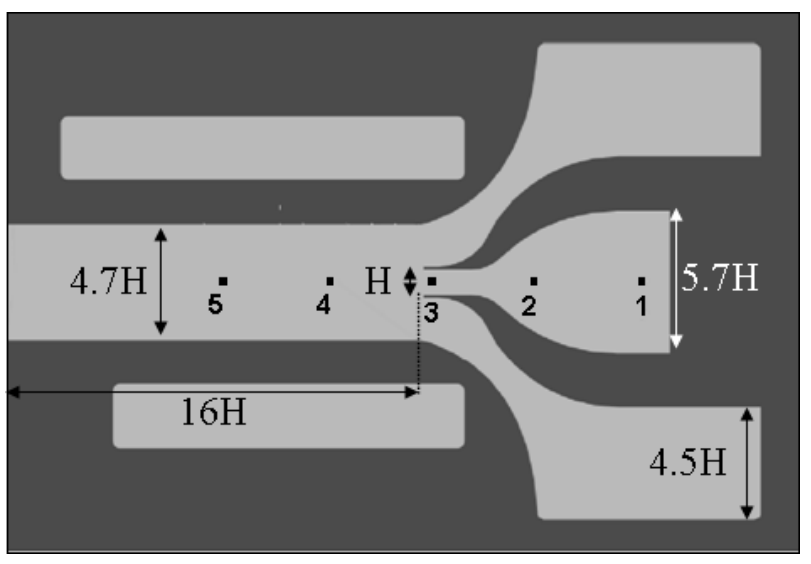

Figure 5. Nozzle used for current study, where $\mathbf{H}=\mathbf{9 . 6} \mathbf{m m}$ (numbers correspond to transducer tappings). 


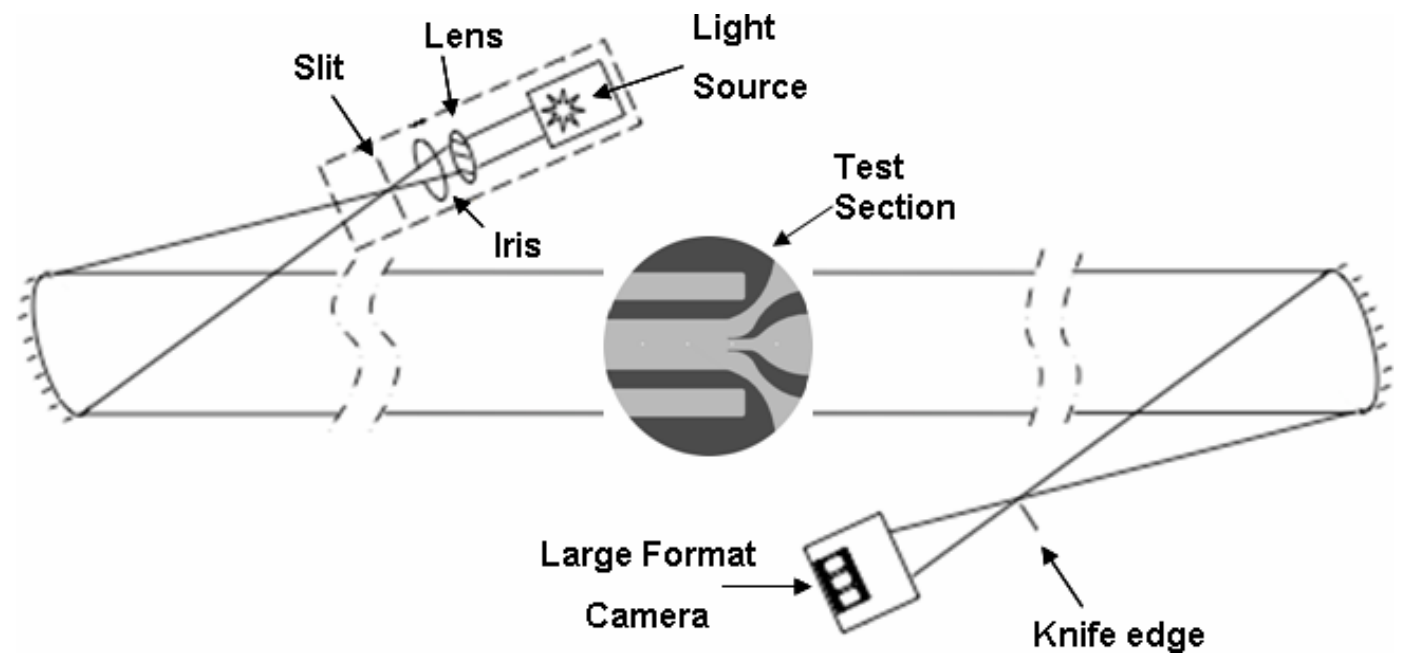

Figure 6. Schematic of the schlieren setup.

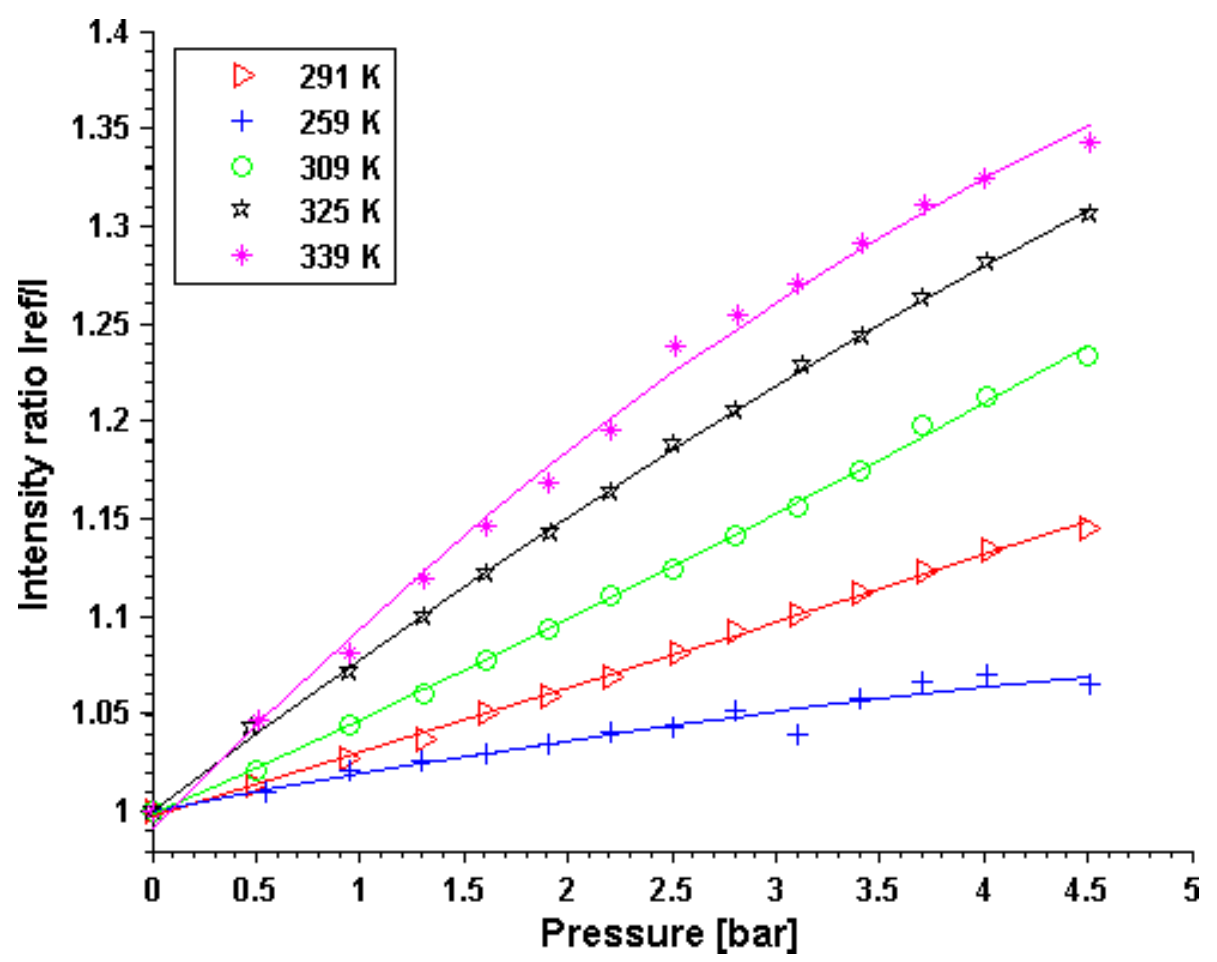

Figure 7. Stern-Volmer plot with second order polynomial curve fitting for PSP2-Ace. 


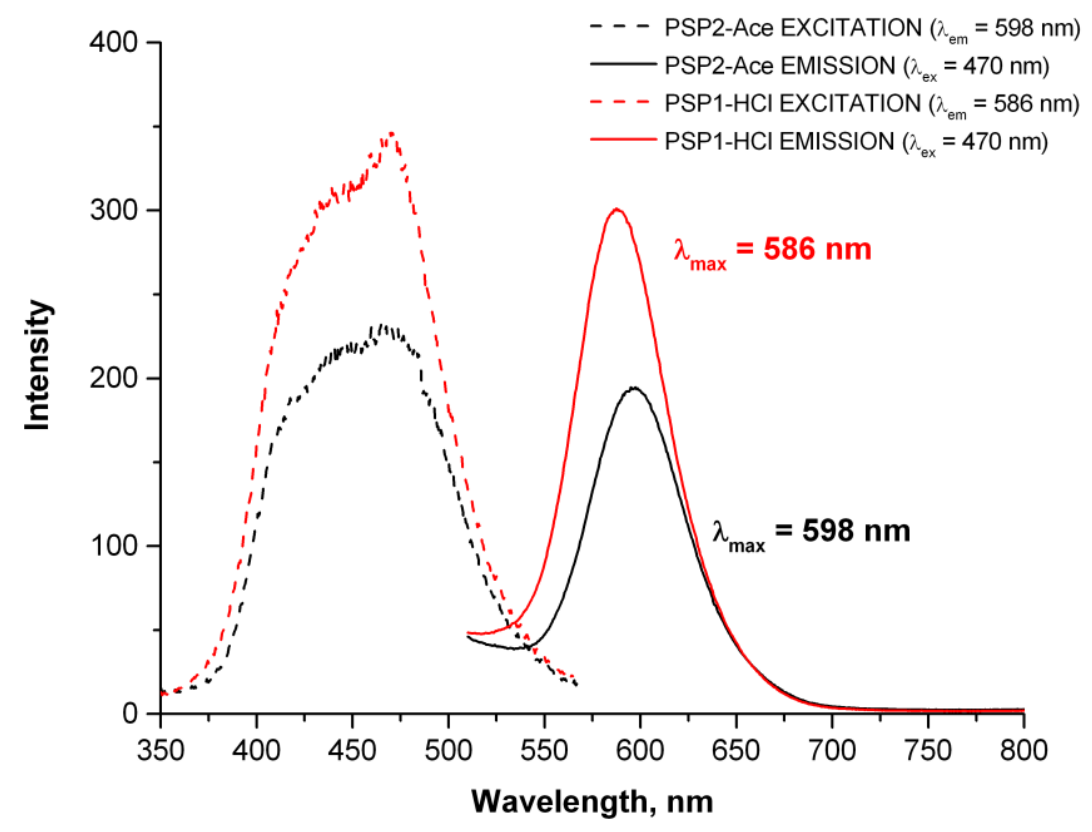

Figure 8. Emission and excitation spectra of the PSP thin films.

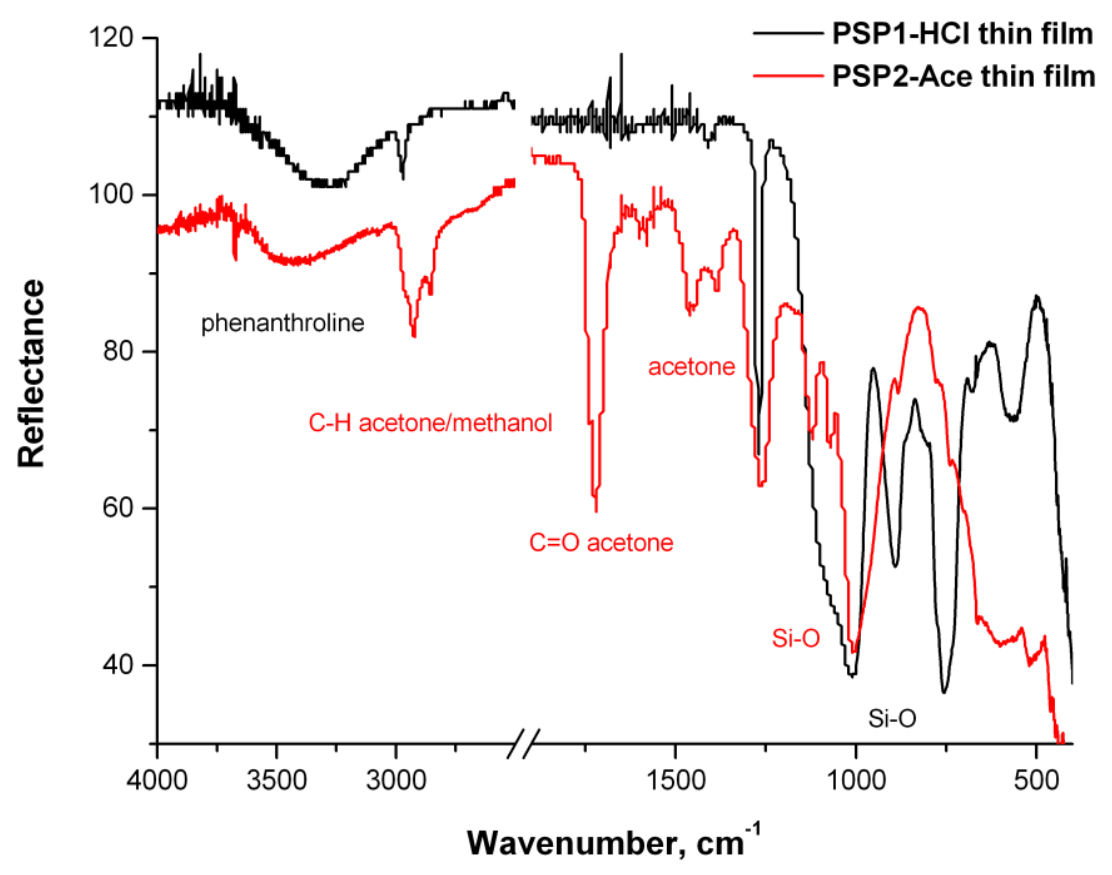

Figure 9. Attenuated total reflection Fourier-transform infrared spectroscopy analysis of the PSP thin films. 

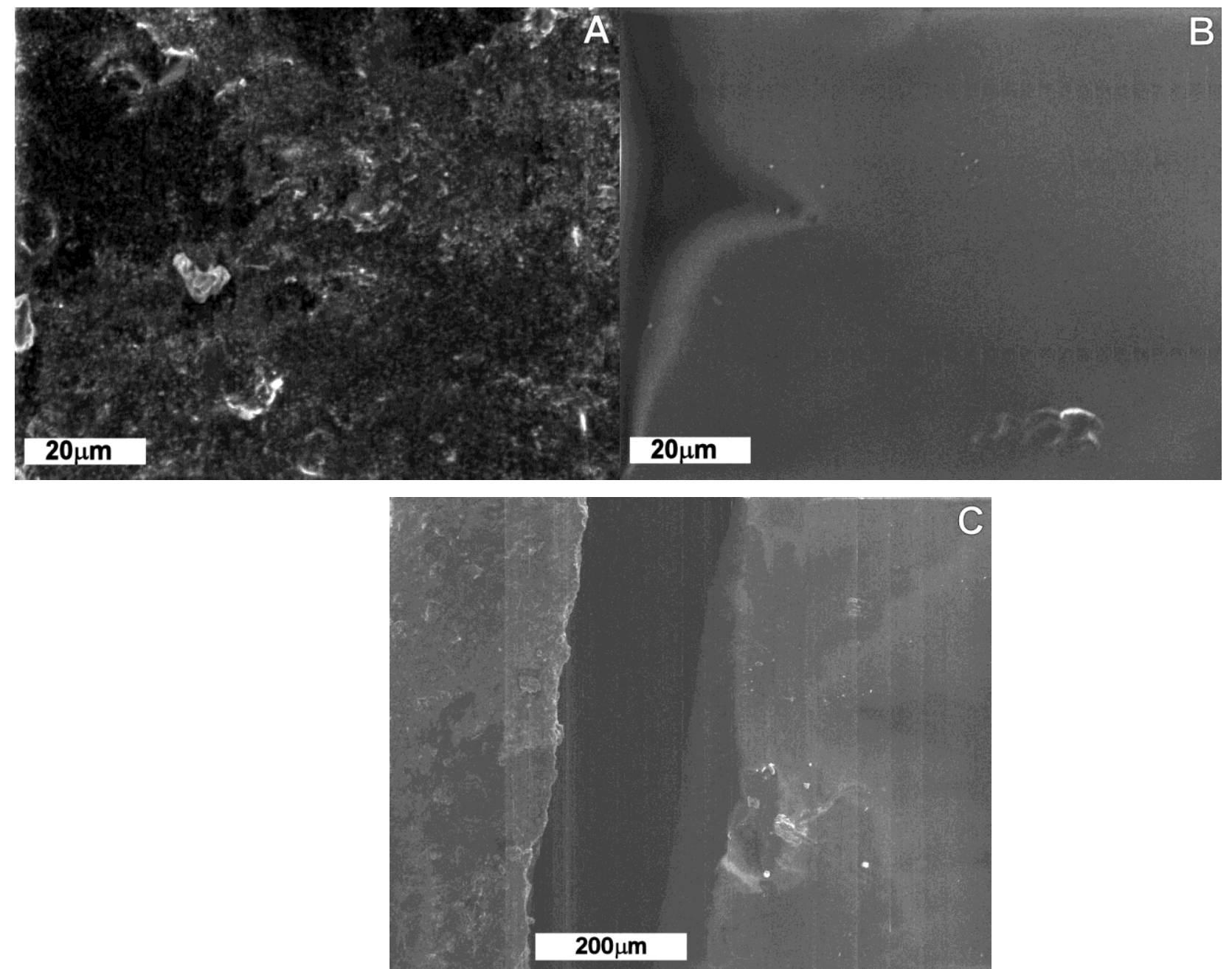

Figure 10. SEM images of (a) PSP2-Ace thin film, (b) PSP1-HCl thin film and (c) comparison of films, PSP2-Ace is to the left of the image and PSP1-HCl is to the right. 


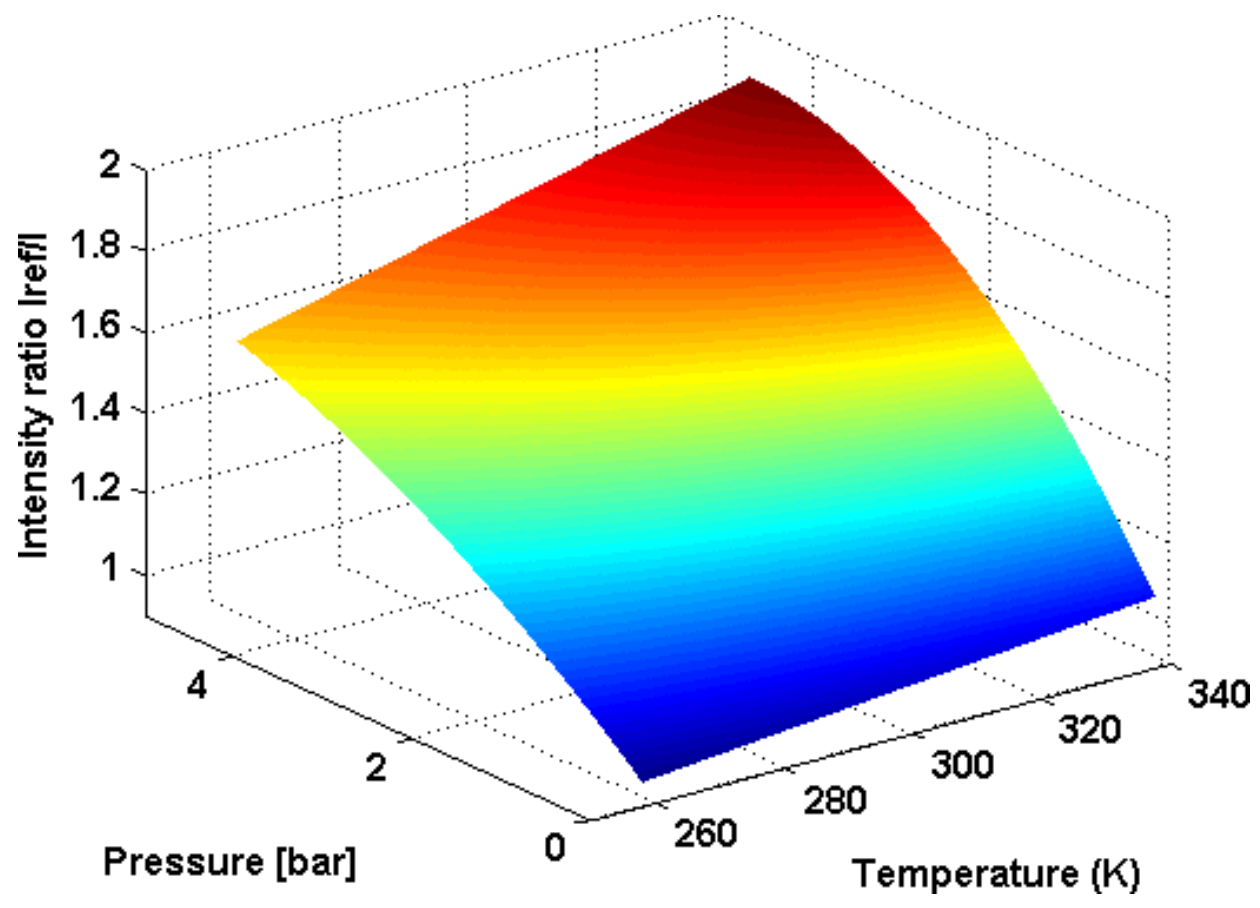

Figure 11. Stern-Volmer plot for PSP1-HCL.

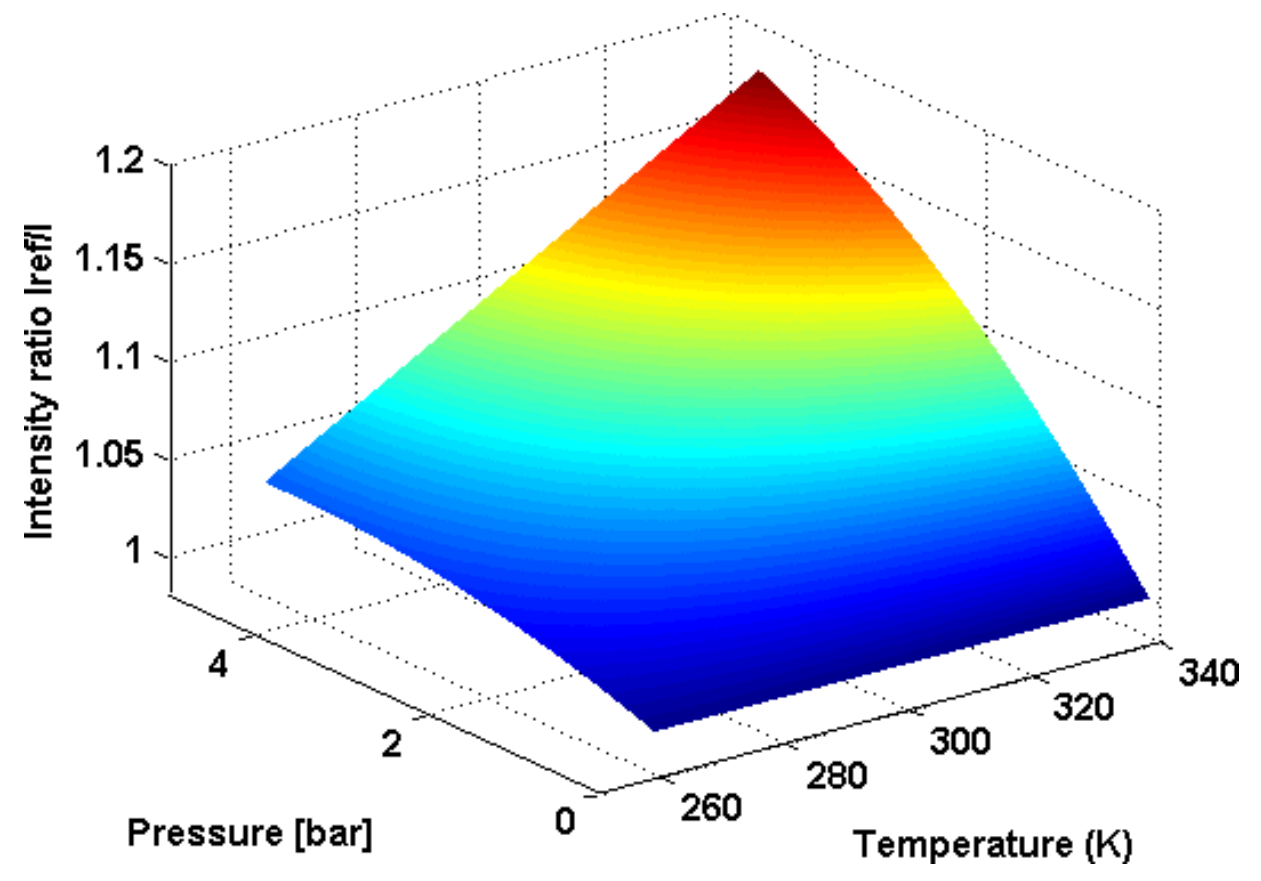

Figure 12. Stern-Volmer plot for PSP2-Ace. 


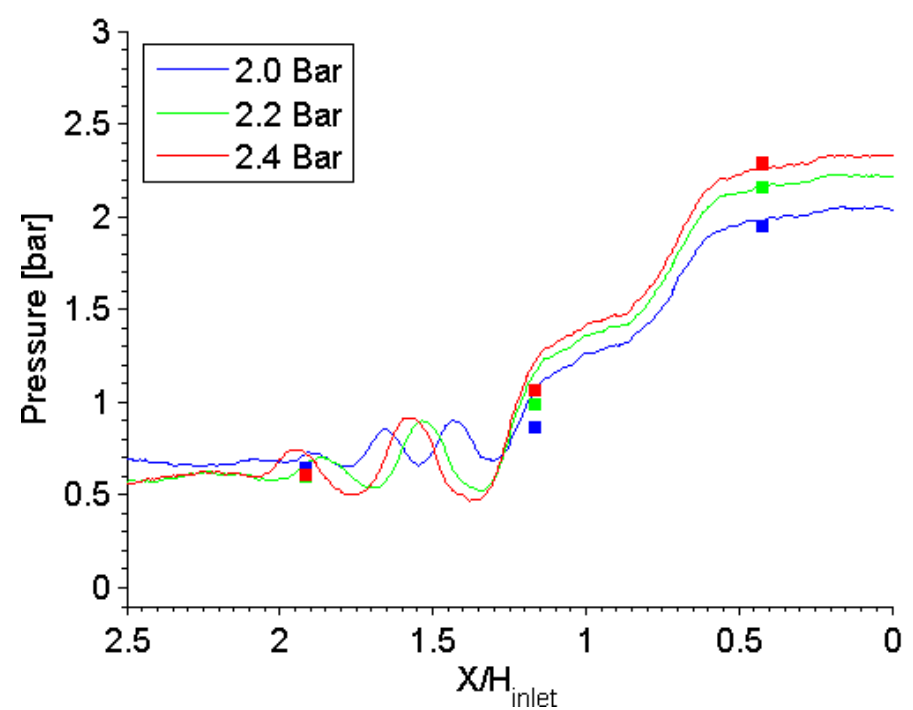

(a)

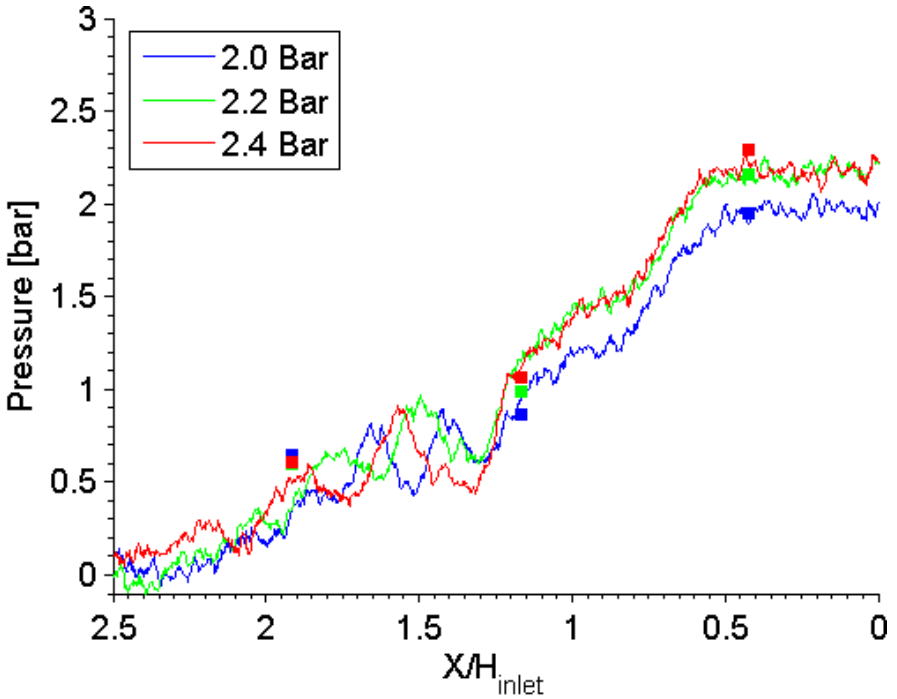

(b)

Figure 13. Comparison of (a) PSP1-HCl and (b) PSP2-Ace results with discrete measurements. 


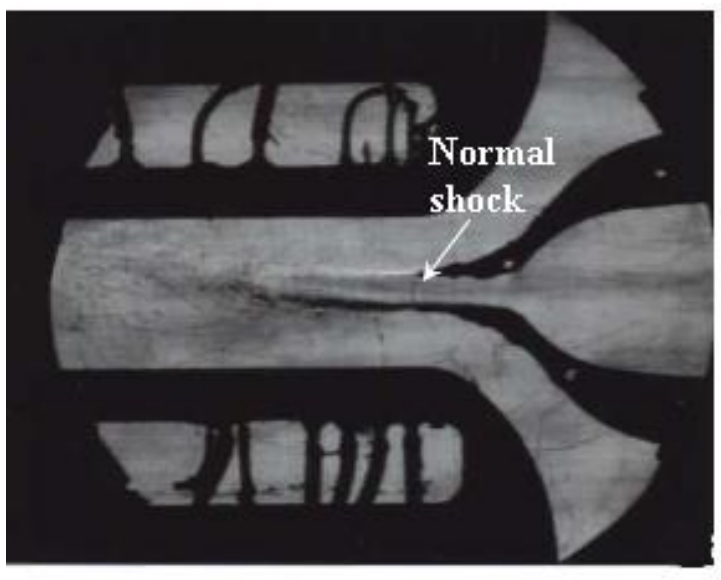

(a)

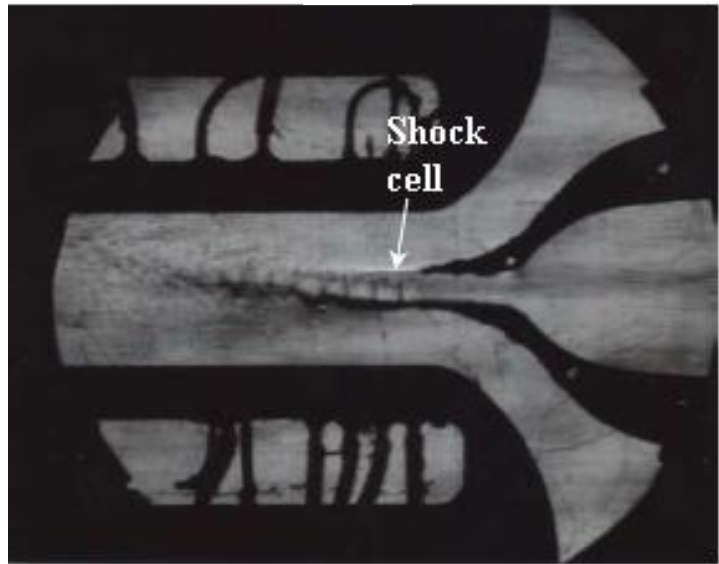

(c)

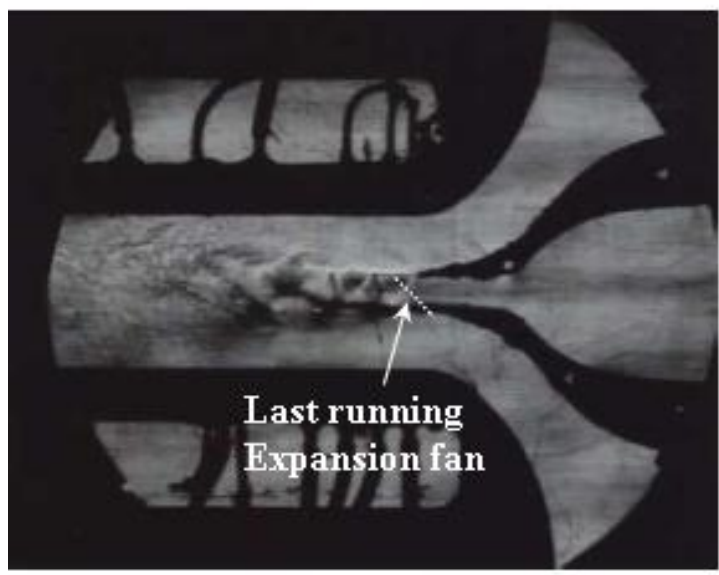

(e)

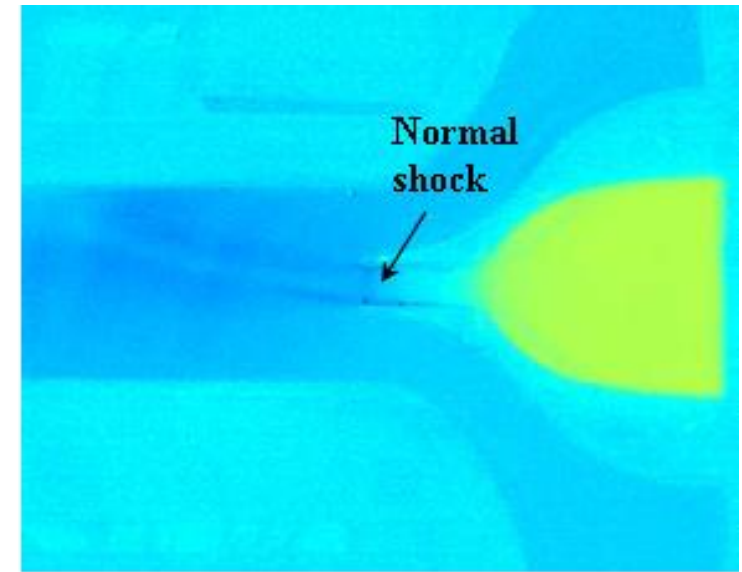

(b)

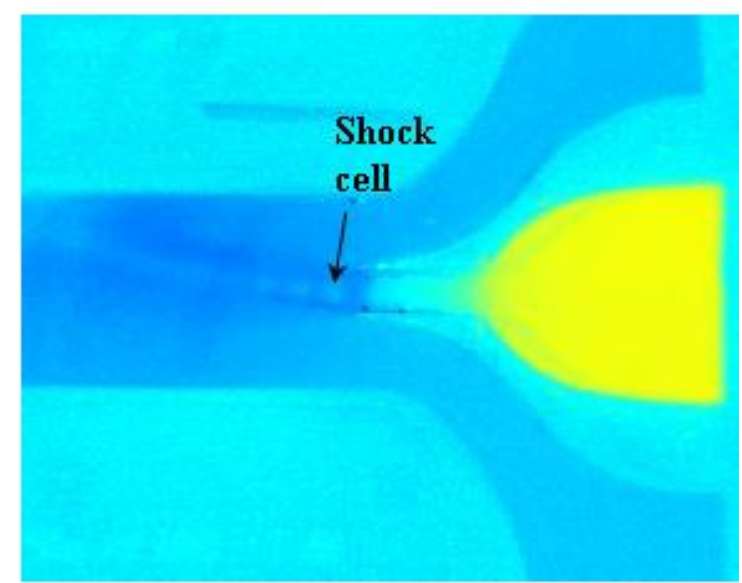

(d)

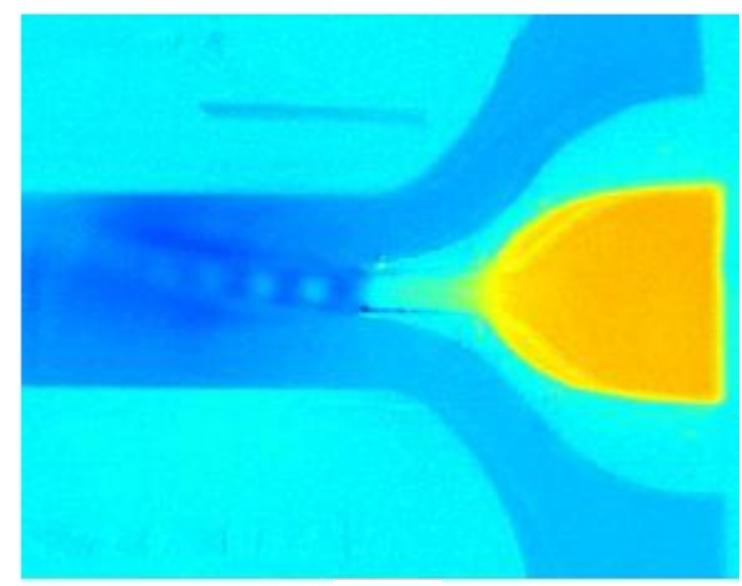

(f)

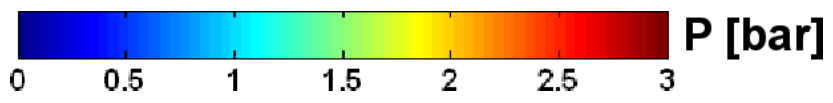

Figure 14. Schlieren and PSP results: (a) \& (b) $P_{\text {inlet }}=1.6$ bar, (c) \& (d) $P_{\text {inlet }}=1.8$ bar, (e) \& (f) $P_{\text {inlet }}=2.0$ bar. 


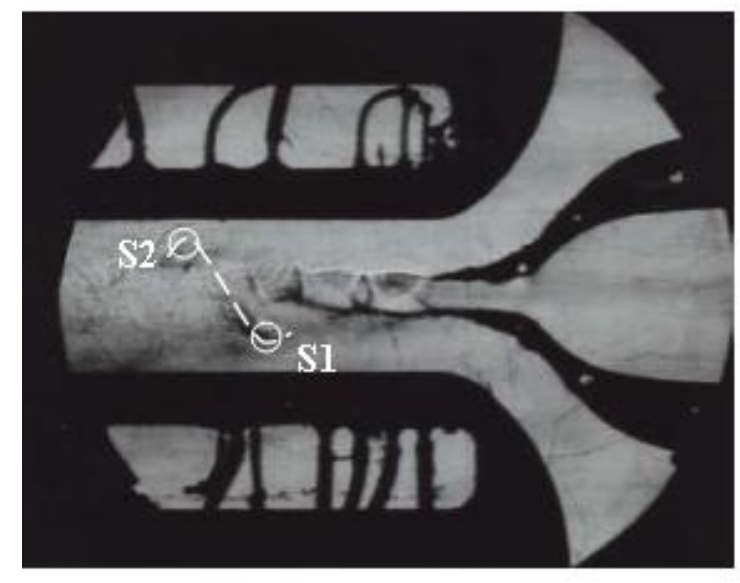

(a)

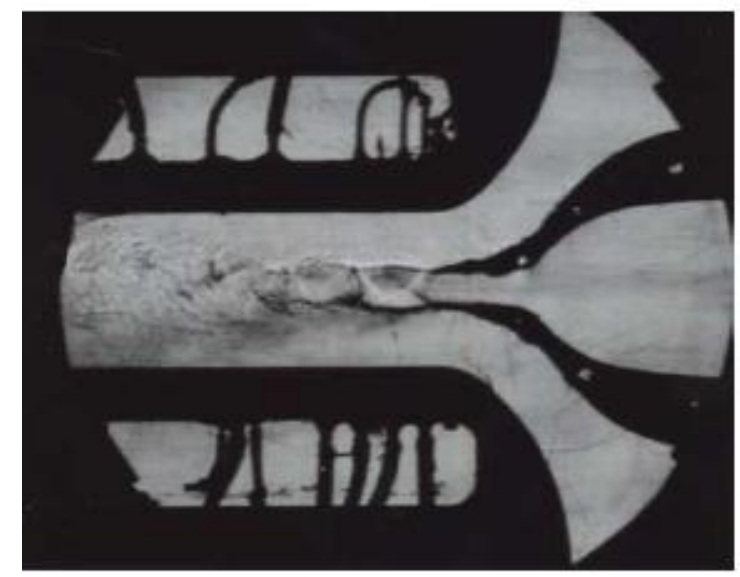

(c)

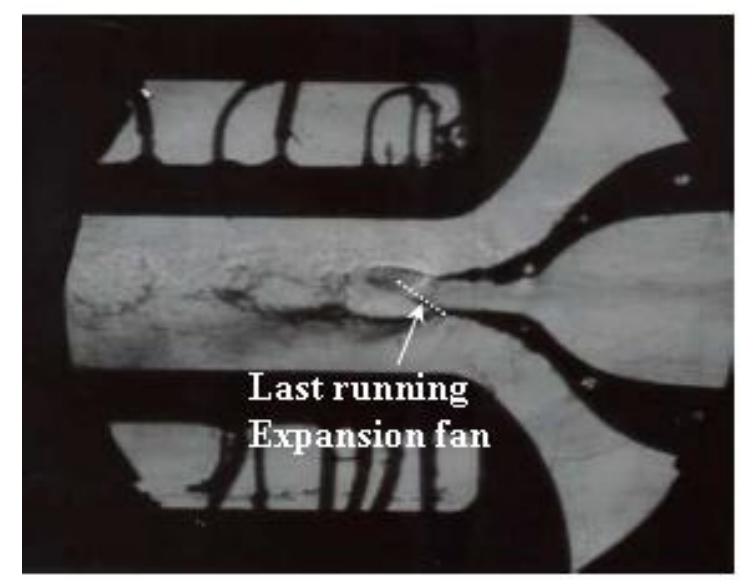

(e)

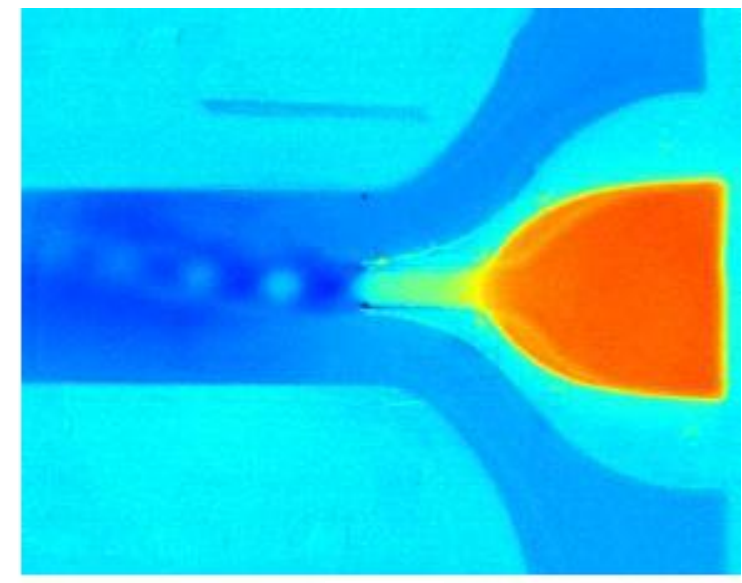

(b)

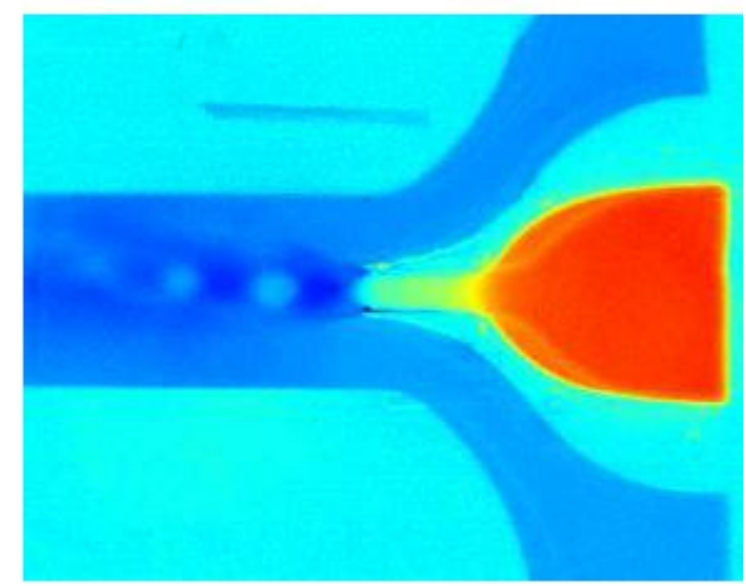

(d)

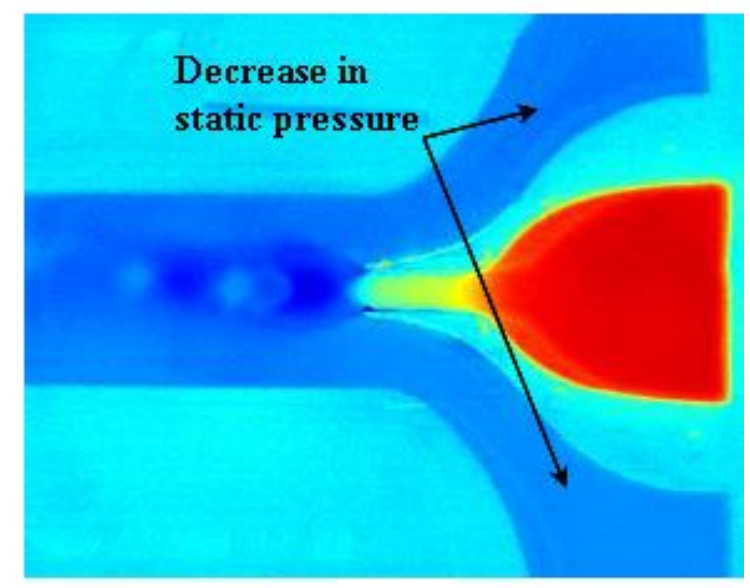

(f)

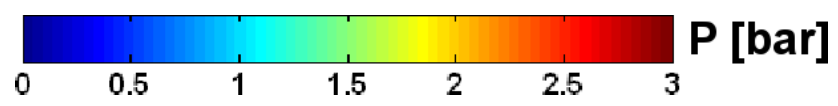

Figure 15. Schlieren and PSP results: (a) \& (b) $P_{\text {inlet }}=2.40$ bar, (c) \& (d) $P_{\text {inlet }}=2.60$ bar, (e) \& (f) $P_{\text {inlet }}=3.0$ bar. 


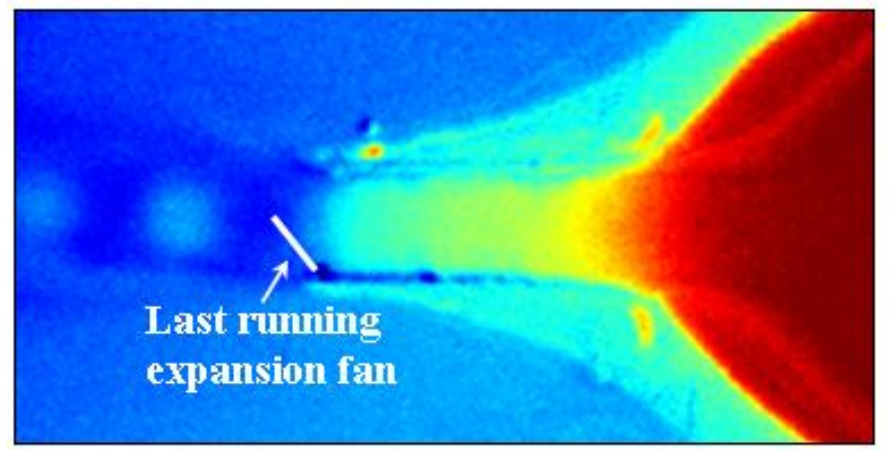

(a)

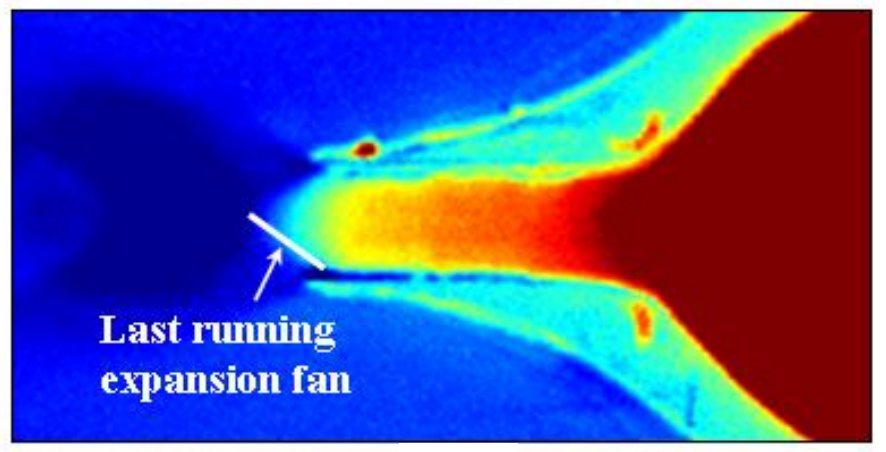

(b)

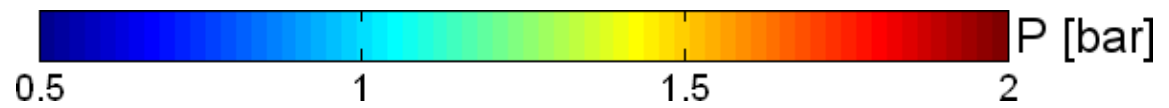

Figure 16. Close-ups of PSP results corresponding to inlet pressure $P_{\text {inlet }}=$ (a) 2.0 bar, (b) 3.0 bar (similar to Figures 14 (e) \& (f) and Figures 15(e) \& (f)).

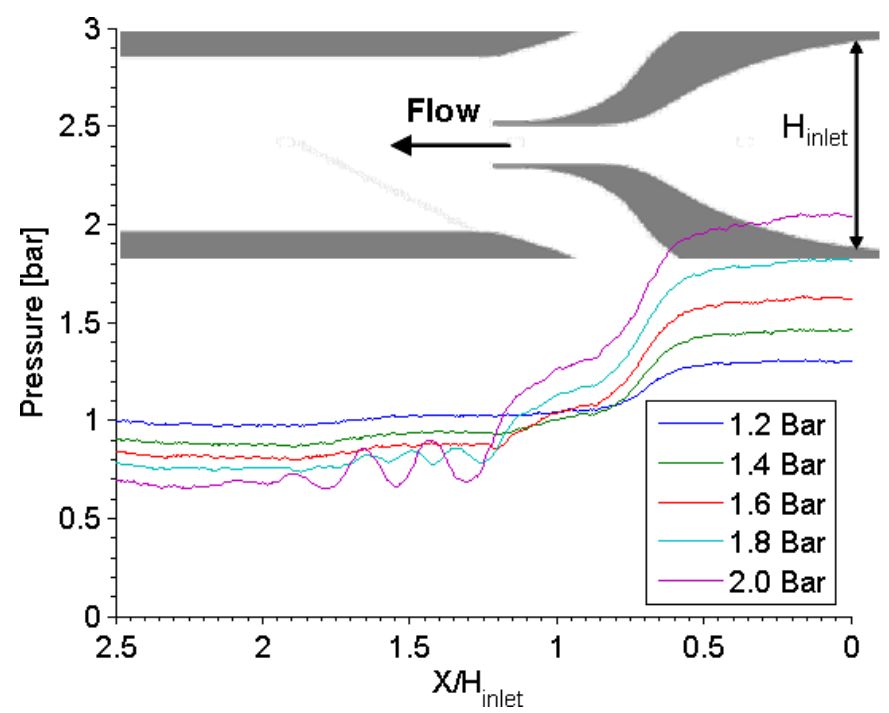

(a)

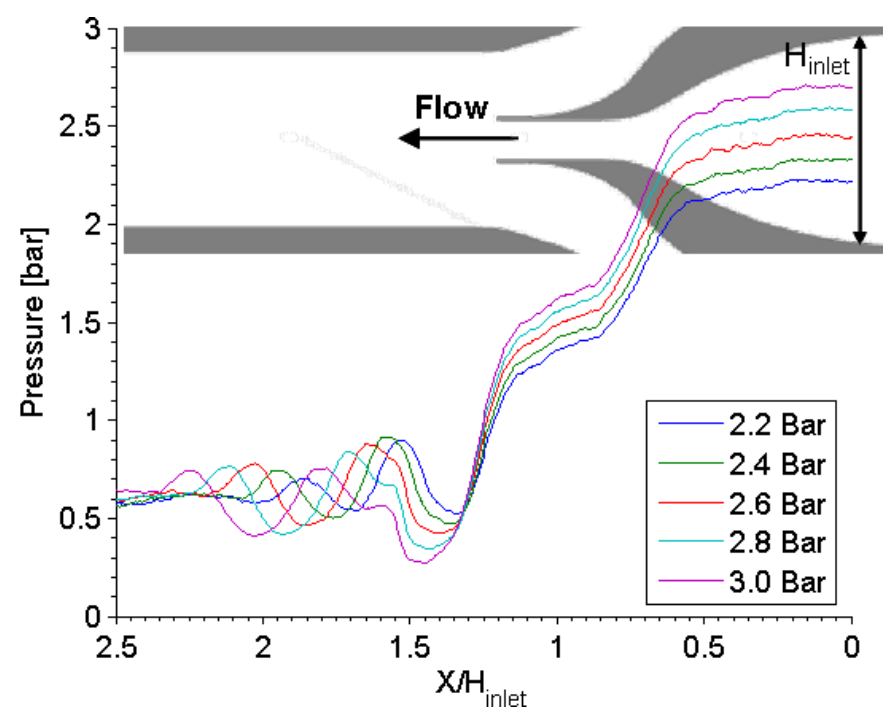

(b)

Figure 17. Pressure profiles along the central axis of the nozzle at various inlet pressures using PSP1-HCl. 


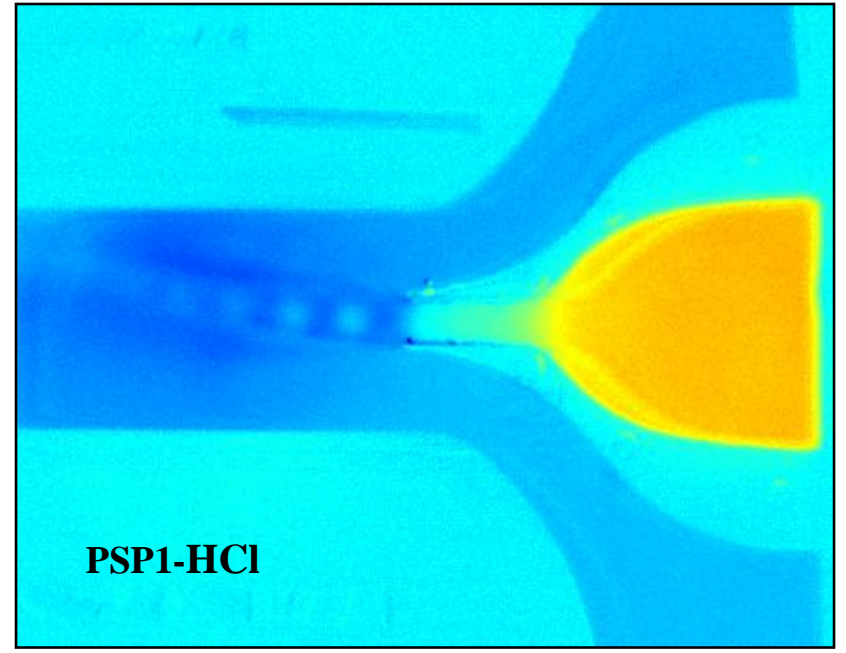

(a)

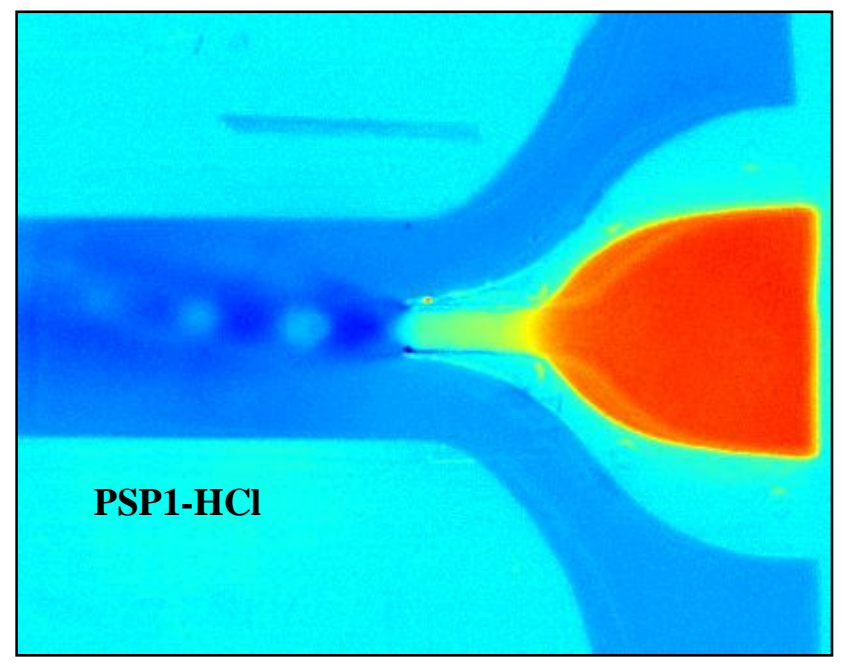

(c)

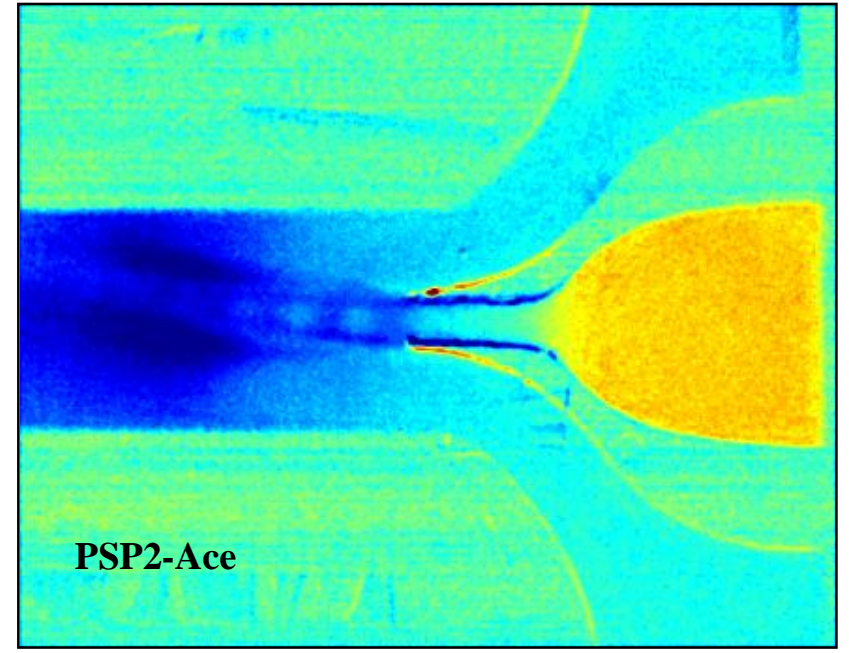

(b)

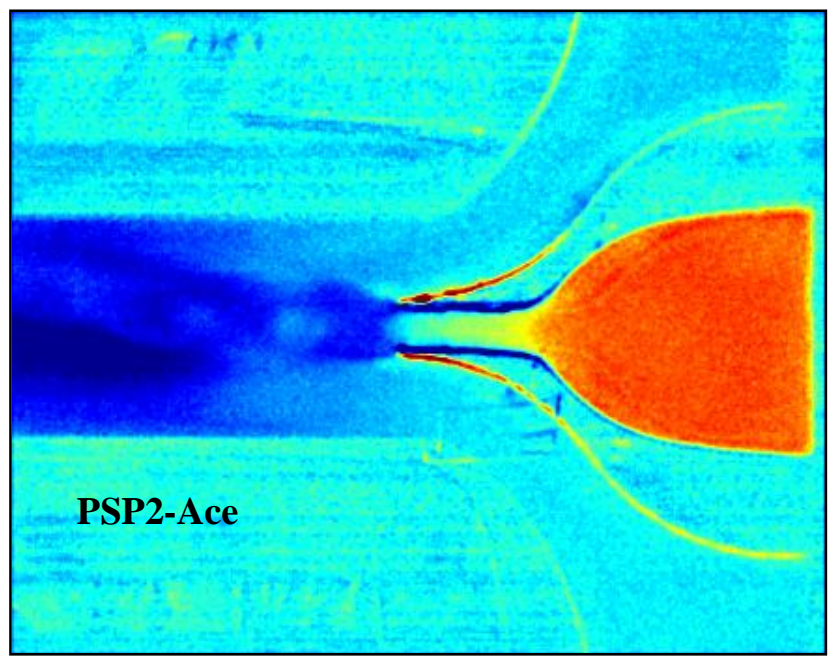

(d)

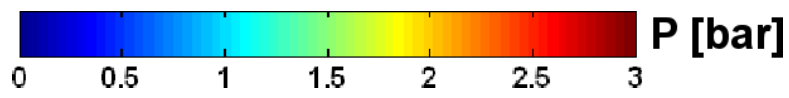

Figure 18. Comparison between PSP1-HCl and PSP2-Ace, (a) \& (b) $P_{\text {inlet }}=2.0 \mathrm{bar}$, (c) \& (d) $P_{\text {inlet }}=2.6$ bar 